\title{
Effects of Tropospheric Wind Shear on the Spectrum of Convectively Generated Gravity Waves
}

\author{
JADWIGA H. BERES \\ University of Washington, Seattle, Washington \\ M. JoAn AleXANDER \\ Colorado Research Associates, Boulder, Colorado \\ JAMES R. HOLTON \\ University of Washington, Seattle, Washington
}

(Manuscript received 22 May 2000, in final form 25 October 2001)

ABSTRACT

\begin{abstract}
The authors examine the effects of tropospheric wind shear on the phase speed spectrum of gravity waves generated by tropical convection. A two-dimensional cloud-resolving model is used to perform numerous squall line simulations with the vertical shear of the horizontal wind varied in three layers of the troposphere. Several simplified simulations using prescribed heating are also performed to elucidate the interactions of wind shear with thermal forcing. It is found that the dominant phase speed range of convectively generated stratospheric gravity waves is primarily determined by the vertical scale of the tropospheric heating and is then modified by the tropospheric wind. The gravity wave spectrum is especially sensitive to shear in the upper troposphere. Through a mechanism similar to critical level filtering, such shear acts to reduce the momentum flux of waves propagating in the same direction as the storm-relative mean wind. Through interaction with convective turrets, shear in the upper troposphere increases the momentum flux of waves propagating opposite to the storm-relative mean wind (the "obstacle effect"). The resulting spectrum of momentum fluxes produced by convectively generated gravity waves is generally not symmetric in the east and west directions; the east-west asymmetry depends primarily on the difference between the wind above the storm and the storm's motion. Thus, it is important that the effects of tropospheric wind shear be included in any attempt to parameterize the effects of gravity wave stress and turbulence in general circulation models.
\end{abstract}

\section{Introduction}

The quasi-biennial oscillation (QBO) and the semiannual oscillation (SAO) of the equatorial middle atmosphere are dramatic examples of low-frequency oscillations produced by wave mean-flow interaction. According to the traditional theory of Holton and Lindzen (1972) the QBO is driven primarily by large-scale equatorial Kelvin and Rossby gravity waves. These modes, which are thought to be generated in the troposphere by large-scale convective systems, propagate vertically into the stratosphere. Qualitatively, these two modes can account for the alternating eastward and westward shear layer descent of the observed QBO. Model studies (e.g., Takahashi and Boville 1992) indicate, however, that the observed Kelvin and Rossby-gravity waves are too

Corresponding author address: Jadwiga H. Beres, Department of Atmospheric Sciences, BOX 351640, University of Washington, Seattle, WA 98195.

E-mail: beres@atmos.washington.edu weak to completely account for the observed zonal accelerations in the QBO. Recently, Dunkerton (1997) has suggested that additional forcing of these stratospheric oscillations can be provided by smaller scale, convectively generated gravity waves that break at their critical levels and transfer momentum to the mean flow. In a modeling study, Alexander and Holton (1997, hereafter AH97) estimated that small-scale convectively generated gravity waves could provide up to $25 \%$ of the forcing required to drive the QBO. Similar estimates have been made by Piani et al. (2000). Kelvin waves are believed to play a major role in forcing the eastward phase of the SAO (Hitchman and Leovy 1988; Canziani et al. 1995), however these waves can not account for all the required forcing. Significant contributions of gravity wave forcing to the SAO were suggested by Ray et al. (1998).

Small horizontal wavelength $(10-200 \mathrm{~km})$ and short period (10-100 min) gravity waves generated by convection are not resolved in global circulations models 
(GCMs), thus their effects on the resolved flow must be parameterized. Forcing caused by convectively generated gravity waves is difficult to parameterize because it is not known how to relate the gravity wave forcing to resolved dynamical fields in the GCMs. The temporal and spatial variability of the convective sources is poorly known, and the processes by which gravity waves are generated by convective disturbances are not clear.

The temporal and spatial variability of vertically propagating convectively generated gravity waves is poorly known because all of the currently available observational techniques have serious limitations in either time or space. Radar observations have high time resolution, but are limited to only a few locations on the globe (e.g., Sato 1992, 1993). Radiosondes have good spatial coverage over land areas, but poor time resolution, and can only be used to indirectly deduce the characteristics of short vertical wavelength gravity waves (Allen and Vincent 1995; Vincent and Alexander 2000). Satellite radiance data has been used to map the global distribution of gravity wave variances at several levels in the stratosphere by Wu and Waters (1996). However, their method is only capable of resolving very long vertical wavelength gravity waves, and must be used with great caution in deduction of regional and seasonal variations in wave sources (Alexander 1998; McLandress et al. 2000). Aircraft campaigns provide the highest horizontal resolution observations of wave activity; however, they provide little information on the vertical structure of waves and are limited to small areas (Pfister et al. 1993; Alexander and Pfister 1995; Alexander et al. 2000). All of the above observational methods have linked substantial gravity wave activity to convection, however none of them provide adequate information to parameterize the full spectrum of convectively generated waves and their sources.

The second difficulty associated with gravity wave parameterization is related to the incomplete understanding of the mechanisms that generate gravity waves. There are currently three theories of wave generation by convection.

1) Mechanical oscillator. Clark et al. (1986) proposed that oscillating updrafts and downdrafts vertically displace the isentropes at the base of a stable layer, for example the stratosphere, producing vertically propagating gravity waves in a manner similar to that of a mechanical oscillator in a stratified fluid. The frequency of waves generated in this fashion is specified by the frequency of the oscillator, or in the case of convection, by the frequency of convective updrafts and downdrafts. Squall line simulations by Fovell et al. (1992), Alexander et al. (1995, hereafter AHD), and Lane et al. (2001) showed evidence for this type of wave generation.

2) Deep heating. In this mechanism thermal forcing by latent heat release within the convective storm is the main gravity wave forcing. Through a number of numerical experiments, Salby and Garcia (1987) showed that the dominant tropospheric vertical wavelength of waves generated by a thermal forcing is approximately equal to twice the vertical extent of the heating. For a fixed frequency and horizontal scale, the vertical wavelength varies inversely with the buoyancy frequency. Since the buoyancy frequency in the stratosphere is approximately double that in the troposphere, the vertical wavelength of stratospheric waves predicted by this mechanism should be equal to the vertical extent of the tropospheric heating. This dependence of vertical wavelength of gravity waves on the depth of the heating region has been confirmed by observations (McLandress et al. 2000) as well as by other numerical studies of convection (AHD; Pandya and Alexander 1999).

3) Obstacle effect. In this mechanism, also known as the moving or transient mountain effect, the convective element acts as a barrier to the background flow, producing upstream propagating waves in a manner similar to flow over a mountain (Pfister et al. 1993). Since the convective element oscillates in time and may move relative to the ground, the generated waves are not stationary relative to the ground as in the case of mountain waves, but propagate horizontally, primarily opposite to the wind direction (Pfister et al. 1993). A detailed study of gravity waves observed over the tropical Indian Ocean confirms this type of wave generation (Alexander and Vincent 2000).

A considerable complication in determining which of these mechanisms dominates in wave generation is associated with the transience of convective forcings. Two- and three-dimensional modeling studies of gravity waves generated by squall lines (Fovell et al. 1992; AHD; AH97; Piani et al. 2000; Lane et al. 2001) show not only a great asymmetry in wave propagation between the eastward and westward directions, but also reveal varying wave properties with the time evolution of convection. Animations of convective storm simulations reveal that stratospheric waves are strongly linked to the storm dynamics. One of the main environmental factors affecting the storm structure is tropospheric wind. The main objective of this paper is to elucidate the effects of tropospheric wind shear on wave generation and propagation to the stratosphere through numerical simulations of tropical squall lines.

Tropical squall lines are observed to have a variety of orientations. For simplicity we assume that the squall lines are oriented in a north-south direction. Weak meridional winds and strong vertical shear of the zonal wind at low levels are characteristic of north-southoriented tropical squall line environments. Since squall lines are long-lasting vigorous convective storms, they provide a good source of gravity waves. Furthermore, 
squall lines can be modeled fairly accurately using a two-dimensional model (Rotunno et al. 1988). Preliminary comparisons with three-dimensional simulations showed that the asymmetry between eastward and westward propagating waves generated by a three-dimensional squall line is reproduced well in two-dimensional simulations, as long as the meridional winds are weak. Since variations of the zonal wind profile are the subject of this study, all simulations will be carried out using a two-dimensional model. This is computationally more efficient and allows a large number of simulations to be carried out.

The goals of this paper are to examine the effects of tropospheric wind shear on the mechanisms responsible for wave generation and to assess the implications for gravity wave parameterization in GCMs. These objectives are addressed using two approaches. Initially squall line simulations are performed with varied zonal wind in three layers of the troposphere. Second, simplified simulations using specified heating are carried out to clarify the interactions between thermal forcing and wind shear. Last, it is shown how wind shear can be responsible for the temporal variability of wave activity that is found both in observations and modeling studies.

\section{Model description}

All simulations in the study are carried out using a variant of the Durran and Klemp (1983) cloud-resolving model similar to that described in Skamarock and Klemp (1993), and identical to that used in Piani et al. (2000). This is essentially the same model that was used previously by Fovell et al. (1992), AHD, and AH97 in twodimensional squall line simulations. Model equations are listed in Durran and Klemp (1983) and in Piani et al. (2000).

In the current study, the domain size is $1200 \mathrm{~km}$ in the horizontal and $30 \mathrm{~km}$ in the vertical with resolution of $1500 \mathrm{~m}$ and $250 \mathrm{~m}$, respectively. The large horizontal domain is used to minimize the propagation of gravity waves out of the model domain through the lateral boundaries. An open wave permeable, lateral boundary condition with gravity wave outflow phase speed of $30 \mathrm{~m} \mathrm{~s}^{-1}$ is used as described in Fovell et al. (1992). A radiation condition is employed at the top boundary (Durran 1999, 427-431). A fourth-order horizontal diffusion scheme is applied to all the variables to avoid nonlinear instabilities. Richardson numberdependent subgrid-scale mixing is responsible for wave damping in strong shear and wave dissipation near critical levels.

\section{Squall line simulations}

\section{a. Initial conditions}

The thermodynamic sounding used to initialize all simulations is the same as in AH97 and is representative

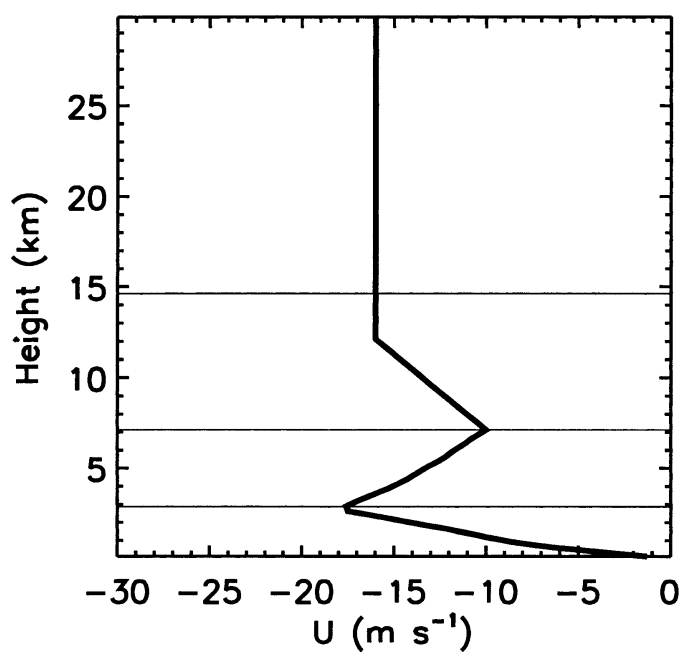

FIG. 1. Initial zonal wind profile relative to the ground used to initialize the basic case simulation (in $\mathrm{m} \mathrm{s}^{-1}$ ). The thin horizontal lines mark the three regions of the troposphere in which variations of shear will be performed.

of a West African squall line. This sounding was slightly modified from observations because, in order to obtain sustained convection in a two-dimensional model, the sounding must be conditionally unstable and have large values of convective available potential energy (CAPE).

Model simulations were initialized using both a warm bubble and a cold pool to make sure that the results were not influenced by initialization methods. For both types of initialization, the minimum temperature perturbation required to produce sustained convection was used, $3 \mathrm{~K}$ for the warm bubble and $-6 \mathrm{~K}$ for the cold pool. For consistency, results from simulations initialized with a cold pool are presented in this paper.

Each simulation in this study is initialized with a different zonal wind profile. Figure 1 depicts a piecewise linear version of the wind profile observed during the West African squall line. This profile is used to initialize the basic case simulation and serves as a guide for further wind profile variations. In other words, the zonal wind profiles that are used to initialize the model in subsequent simulations are reasonable representations of the tropical environment. Wind shear is varied in the three regions of the troposphere: low, middle and upper, depicted in Fig. 1. There is no shear in the stratosphere, above $15 \mathrm{~km}$, since the full spectrum of waves generated in the troposphere and entering the stratosphere is of main interest in this study. Details of wind shear variations in the three tropospheric layers are presented in sections $3 \mathrm{c}$ and $3 \mathrm{~d}$.

The model domain translation speed in each simulation is set to the mean storm propagation speed. The propagation speed of a squall line is determined by the low level jet (Rotunno et al. 1988). More specifically, squall line propagation speed is approximately equal to the horizontal wind strength at $\sim 2.5 \mathrm{~km}$. For example, the storm in the basic case moves to the west on average 
a) Developing Stage

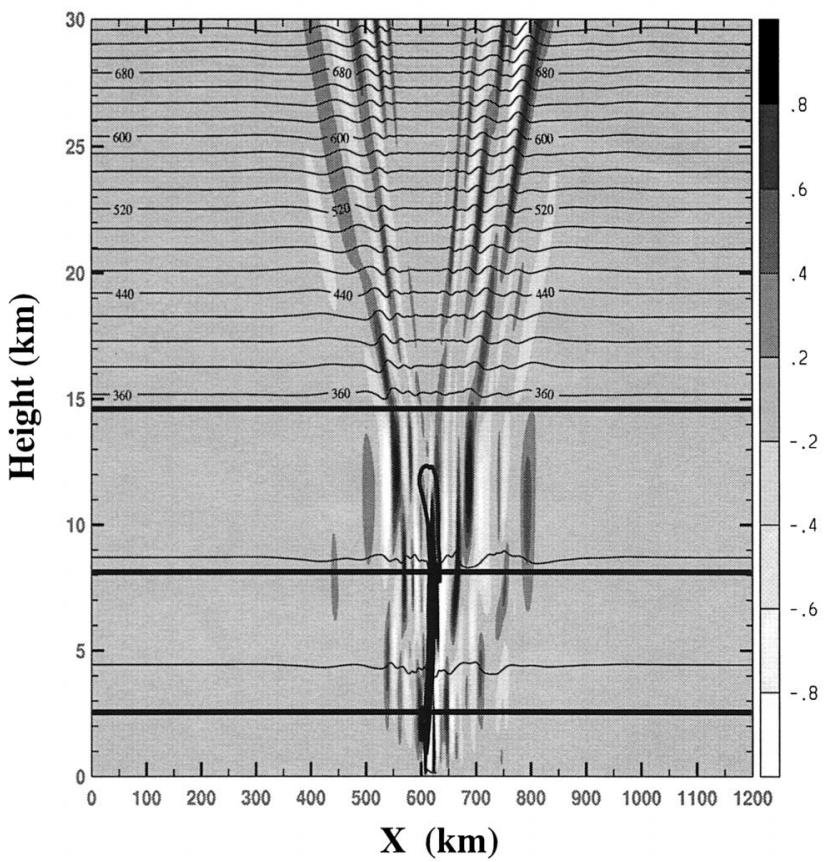

b) Mature Stage

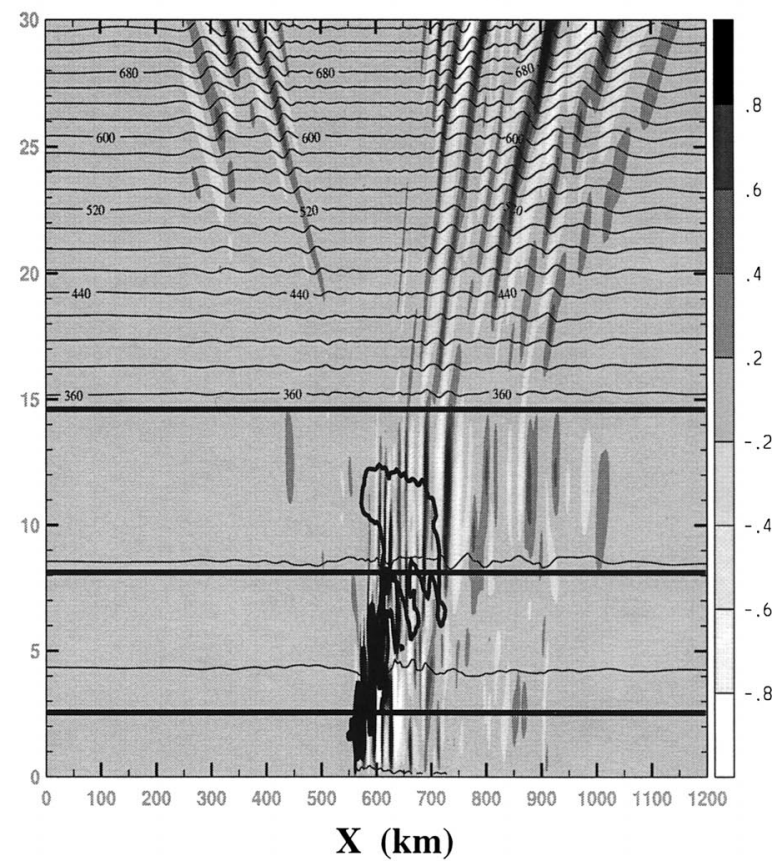

FIG. 2. The basic case simulation initialized with a cold pool at (a) $t=2.5 \mathrm{~h}$ (developing stage) and (b) $t=5 \mathrm{~h}$ (mature stage 1 ). Shading represents vertical velocity perturbations of magnitude less than $1 \mathrm{~m} \mathrm{~s}^{-1}$. Thin lines represent isentropes at $20-\mathrm{K}$ intervals. Thick black solid line shows the cloud outline (cloud water mixing ratio $=10^{-4} \mathrm{~g} \mathrm{~g}^{-1}$ ). Shaded black region is the region of most intense latent heat release. Thick horizontal lines mark the three regions of the troposphere in which variations of shear will be performed.

at $16 \mathrm{~m} \mathrm{~s}^{-1}$. This method of domain translation keeps the storm near the center of the domain. Therefore, the areas in which convectively generated waves are observed are nearly equal on both sides of the storm. This allows objective viewing of eastward and westward propagating waves.

\section{b. Methods of wave assessment}

Stratospheric vertical flux of horizontal momentum is chosen to be the primary measure of gravity wave activity since this quantity directly influences the dynamics of the middle atmosphere; the vertical divergence of momentum flux is proportional to the zonal force exerted on the mean flow by gravity waves. The use of momentum flux as a diagnostic quantity is also advantageous since considering only vertical velocity perturbations tends to emphasize the most vertically propagating waves, whereas considering only zonal wind perturbations emphasizes the most horizontally propagating waves.

As noted in the introduction, the stratospheric gravity wave field varies in time. Figure 2 shows snapshots of the model output at time $t=2.5 \mathrm{~h}$ and $t=5 \mathrm{~h}$ for the basic case simulation. Stratospheric gravity waves at the earlier time are fairly symmetric, whereas at the later time the wave activity to the east of the storm is much greater than the wave activity to the west of the storm.
After several hours of the simulation, the wave field is similar to that at $t=5 \mathrm{~h}$.

Since one of the goals of this paper is to examine gravity wave-generation mechanisms we attempt to link the properties of stratospheric gravity waves directly to the properties of tropospheric convection by which they were forced. In order to achieve this task, we analyze wave characteristics separately for three time periods of the storm evolution during which the convection properties are relatively unchanged. The convection properties we consider are cell structure, convection depth, storm's convective activity, and minimum subcloud buoyancy.

Convective activity (hereafter CA) is defined as a function of time, $t$ :

$$
\mathrm{CA}(t)=w_{\max }^{2}(t)\left(\mathrm{m}^{2} \mathrm{~s}^{-2}\right),
$$

where $w_{\max }$ is the maximum value of tropospheric vertical velocity at a given time. Minimum subcloud buoyancy, $B_{\min }$ is defined as:

$$
B_{\min }(t)=\min \left(\frac{\theta-\bar{\theta}(z)}{\theta_{0}} g\right)\left(\mathrm{m} \mathrm{s}^{-2}\right)
$$

where $\min ()$ refers to a minimum value in the domain at a given time $t$. Here, $B_{\min }$ is representative of the strength of the storm's cold pool. 
a) Convective Activity

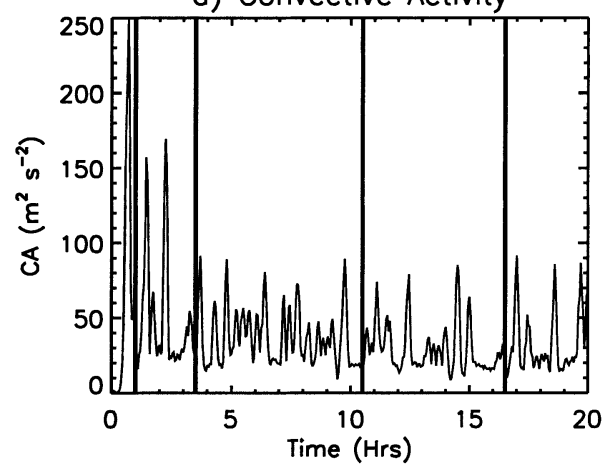

b) Minimum Subcloud Buoyancy

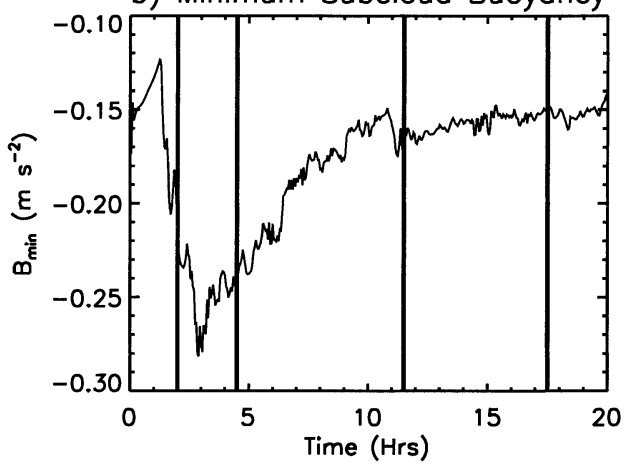

FIG. 3. Time evolution of diagnostics of storm development for the basic case simulation: (a) convective activity (in $\mathrm{m}^{2} \mathrm{~s}^{-2}$ ) and (b) minimum subcloud buoyancy (in $\mathrm{m} \mathrm{s}^{-2}$ ). The solid vertical lines in the figures separate the three stages of storm development (developing, mature 1, and mature 2). The very beginning and end of the simulations are not analyzed.

\section{1) DEFINITION OF STORM's STAGES}

Figure 3 shows the time evolution of convective activity and minimum subcloud buoyancy for the basic case simulation. With the aid of these the storm is subdivided into three stages of development: developing, mature 1 , and mature 2 :

(a)

(b)

(c)

Developing stage. The storm consist of one convective cell, one strong central updraft, and a deep latent heating region ranging up to $10 \mathrm{~km}$ (Fig. 2a). In this stage the magnitudes of vertical velocities are the greatest, ranging up to $12 \mathrm{~m} \mathrm{~s}^{-1}$. The surface cold pool is the strongest during this period, as indicated by the low values of minimum subcloud buoyancy in Fig. 3b. Mature stage 1 . The storm consists of many convective cells propagating rearwards relative to the storm motion and the heating region is relatively shallow, ranging only up to about $7 \mathrm{~km}$ as shown in Fig. $2 b$. In addition, the updrafts are weaker, with maximum vertical velocities of $6 \mathrm{~m}$ $\mathrm{s}^{-1}$ on average. The minimum subcloud buoyancy is rising quickly.

Mature stage 2. Similar to mature stage 1 in structure, however, the cold pool is weaker as compared to mature stage 1 . For the basic case simulation, the two mature stages are essentially the same, but the distinction is clearer in some simulations with altered shear structure.

The stages of storm development defined above are marked by the solid vertical lines in Fig. 3. Note that the three stages are defined at slightly later times when looking at the minimum subcloud buoyancy as compared to the maximum vertical velocity. This is because it takes approximately $1 \mathrm{~h}$ for the precipitation induced by a given strong updraft to reach the surface and evap- orate into the cold pool. The very beginning of the simulations is ignored to ensure that the results do not reflect initialization artifacts. The last $2.5 \mathrm{~h}$ of simulations are also ignored since by that time the storm has propagated quite far from the center of the domain. In summary, with respect to the storm's convective activity, the developing stage is defined between 1 and 3.5 $\mathrm{h}$ of the simulations, mature stage 1 between 3.5 and $10.5 \mathrm{~h}$, and mature stage 2 between 10.5 and $16.5 \mathrm{~h}$.

In this work we attempt to analyze gravity waves generated during the three stages of storm development defined above. This is a difficult task since gravity waves are generated at different levels of the atmosphere and with a wide range of vertical group velocities. An analysis of a gravity wave field during a limited time period cannot perfectly reflect the wave spectrum generated by a convective storm during that time since gravity waves with very low vertical group velocities may not have enough time to propagate to the analysis level and the analysis may include gravity waves generated prior to the analysis period. To minimize the errors in analyzing gravity waves generated during the three stages of development, we perform all momentum flux calculations at the level of $15 \mathrm{~km}$. This minimizes the time that gravity waves travel from their generation region to the analysis level, however the gravity wave spectra at this height are not very smooth, since this level is close to the tropopause.

As will be demonstrated later, the majority of the gravity waves are generated in the heating region in the midtroposphere, well below the analysis level. In order to allow time for those gravity waves to propagate from their generation region to the analysis level, gravity wave activity is measured at the height of $15 \mathrm{~km}$ with $1 \mathrm{~h}$ delay with respect to the stages of storm's development defined by the convective activity. For example, the storm's developing stage is defined between 1 and $3.5 \mathrm{~h}$ with respect to the convective activity. To assess gravity wave activity generated during that time period, 
gravity wave momentum flux is analyzed at $15 \mathrm{~km}$ between 2 and $4.5 \mathrm{~h}$. At $t=2 \mathrm{~h}$ most of the gravity waves that were generated by model initialization have had enough time to propagate above the analysis level, and therefore the calculations presented do not reflect initialization method artifacts. Similarly, gravity wave activity measured at $15 \mathrm{~km}$ between 4.5 and $11.5 \mathrm{~h}$ of the simulation corresponds to mature stage 1 , and gravity wave activity between 11.5 and $17.5 \mathrm{~h}$ of the simulation corresponds to mature stage 2 .

Parameterizations of gravity waves in global circulation models require the knowledge of wave properties during the entire lifetime of a convective storm. For this reason, modeling studies of gravity waves generated by convection often present gravity wave properties averaged over the lifetime of the storm, instead of averages over a short time period. However calculations averaged over the lifetime of the storm are applicable only to the particular modeled storm. Lifetimes of observed tropical squall lines range from several hours to several days. In this work we also present wave characteristics averaged over a short period of convection. This information can be subsequently used to derive quantities integrated over the lifetime of the storm taking into account the length and strength of the convection.

\section{2) Details of calculations}

Momentum flux carried by gravity waves is calculated using the following relation:

$$
F_{m}(k, \omega)=\bar{\rho} \operatorname{Co}\left(u^{\prime} w^{\prime}\right),
$$

where $u^{\prime}$ and $w^{\prime}$ represent the zonal and vertical wind perturbations, $\bar{\rho}$ is the background density, and $\operatorname{Co}\left(u^{\prime} w^{\prime}\right)$ is the cospectrum of $\left(u^{\prime} w^{\prime}\right)$. The cospectrum was calculated using $u^{\prime}$ and $w^{\prime}$ values from the inner $1100 \mathrm{~km}$ of the horizontal domain; the $50 \mathrm{~km}$ region of the horizontal domain near both lateral boundaries is excluded from the average. All calculations are averaged over the time intervals defining the three stages of storm development separately as well as together.

In order to calculate the cospectrum of $\left(u^{\prime} w^{\prime}\right)$, the $u^{\prime}(x, t)$ and $w^{\prime}(x, t)$ arrays are Fourier transformed, yielding $U(k, \omega)$ and $W(k, \omega)$, where $k$ and $\omega$ represent the horizontal wavenumber and frequency. The cospectrum is then given by: $\operatorname{Co}\left(u^{\prime} w^{\prime}\right)=\operatorname{Re}\left(U W^{*}\right)$, where $W^{*}$ is the complex conjugate of $W$. Details of this calculation can be found in AHD.

Equation (3) yields a two-dimensional spectrum of momentum flux as a function of frequency $\omega$ and horizontal wavenumber, $k$. Since the wave energy is upward-propagating, positive momentum flux values correspond to waves propagating eastward, whereas negative flux values correspond to waves propagating westward relative to the mean flow.

The two-dimensional spectrum of momentum flux is used to calculate the following quantities.
1) Domain-averaged eastward and westward momentum flux $\bar{F}_{m}$. Eastward $\bar{F}_{m}$ is defined as the sum of all positive flux values within the $(k, \omega)$ domain, and westward $\bar{F}_{m}$ is defined as the sum of all negative values in the domain.

2) Momentum flux phase speed spectra. Each momentum flux value in the $(\omega, k)$ domain is associated with a phase speed $c=\omega / k$. A phase speed spectrum is produced by grouping all of the $(\omega, k)$ pairs into $2 \mathrm{~m} \mathrm{~s}^{-1}$ phase speed bins, and subsequently summing momentum fluxes in each phase speed bin. In order to obtain a smoother spectrum, the $u^{\prime}(x, t)$ and $w^{\prime}(x, t)$ arrays are padded with zeros in the time domain before Fourier transforming. Zero padding does not add any additional information to the generated spectrum, but only increases its frequency resolution (Percival and Walden 1993). The cospectrum and $F_{m}(\omega, k)$ are then calculated using the padded arrays. Cospectrum values for periods longer than the maximum resolved in the original time series are very small and are discarded. The $F_{m}(\omega$, $k$ ) spectrum is subsequently renormalized so that the fluxes represent average values over the time intervals of the storm's stages. All $(\omega, k)$ pairs in the spectrum are subsequently binned in $c$. This binning procedure yields momentum flux as a function of zonal phase speed, where the integral over all phase speeds equals the domain-averaged momentum flux.

3) Vertical wavelength spectra. For each $(\omega, k)$ pair in the cospectrum, the vertical wavelength of the waves is estimated using the linear gravity wave dispersion relation. The vertical wavelengths obtained are subsequently grouped into $0.5-\mathrm{km}$ bins to obtain a spectrum of momentum flux versus vertical wavelength.

\section{3) StORM-RELATIVE MEAN Wind}

It will be demonstrated below, that a good prognostic of the asymmetry between eastward- and westwardpropagating convectively generated gravity waves is the storm-relative mean wind $\bar{U}_{\mathrm{SR}}$. Throughout this study, $\bar{U}_{\mathrm{SR}}$ is defined as the difference between the wind just above the storm and the storm speed $U_{s}$. In the tropical simulations performed here, the tropopause is located at the $15-\mathrm{km}$ level, which correspond to the maximum height achieved by convective updrafts. Therefore, the storm-relative mean wind $\bar{U}_{\mathrm{SR}}$ is defined as

$$
\bar{U}_{\mathrm{SR}}=\bar{U}_{15 \mathrm{~km}}-U_{s} .
$$

Since the strength of the convection decreases over time and the heating region becomes shallower, the wind speed at $13 \mathrm{~km}$ is sometimes more representative of the wind above the storm, and therefore it is a better predictor of wave structure. For consistency, however, $U_{15 \mathrm{~km}}$ will be used throughout this study in the definition of $\bar{U}_{\mathrm{SR}}$. 
As described in section 3a, the propagation speed of a squall line is determined by the low-level jet. Therefore the storm-relative mean wind $\bar{U}_{\mathrm{SR}}$ can be written approximately as

$$
\bar{U}_{\mathrm{SR}}=\bar{U}_{15 \mathrm{~km}}-\bar{U}_{2.5 \mathrm{~km}} .
$$

\section{c. Variations of upper-level shear}

In order to assess the effects of upper-level tropospheric shear on the wave spectrum, the basic wind profile was altered above $7 \mathrm{~km}$. Because the upper-level shear has little influence on the dynamics of a convective storm, the forcing below $7 \mathrm{~km}$ is very similar in all these simulations. The squall lines produced in these simulation with varying upper-level shear had similar time evolutions, similar amplitudes of updrafts and downdrafts, similar strengths of the cold pool and similar precipitation rates. Despite these similarities in storm structure, there were considerable differences in the gravity waves generated in these simulations. These differences will be quantified by evaluating the momentum flux magnitudes and spectral characteristics of the generated gravity waves.

\section{1) EXPERIMENTAL DESIGN}

Seven simulations were performed with shear varying between 0 and $3.6 \times 10^{-3} \mathrm{~s}^{-1}$ in the layer between 7 and $12 \mathrm{~km}$. The initial wind profiles for these simulations are shown in Fig. 4. Note that winds in this figure are plotted relative to the storm motion, and not relative to the ground. The curve labeled $\bar{U}_{\mathrm{SR}}=0$ corresponds to the basic case. The seven simulations corresponding to the wind profiles shown in Fig. 4 are referred to as the upper-level shear runs.

\section{2) StORM STRUCTURE}

To demonstrate similarities in the storm development for all the simulations with varying upper-level shear, Fig. 5 shows their convective activity averaged over the three stages of storm development, as well as averaged over the three stages of the storm together. The convective activity for the different upper-level shear simulations show small variability. The convective activity is largest in all simulations in the developing stage however the difference between the two mature stages is not always clear. The differences in CA in these seven simulations are quite small considering the highly nonlinear tropospheric dynamics, and should not be responsible for large differences in the amplitudes of generated gravity waves.

\section{3) Momentum fluX Magnitudes}

First, we examine the effects of varied wind shear between 7 and $12 \mathrm{~km}$ on the distribution of the domain-

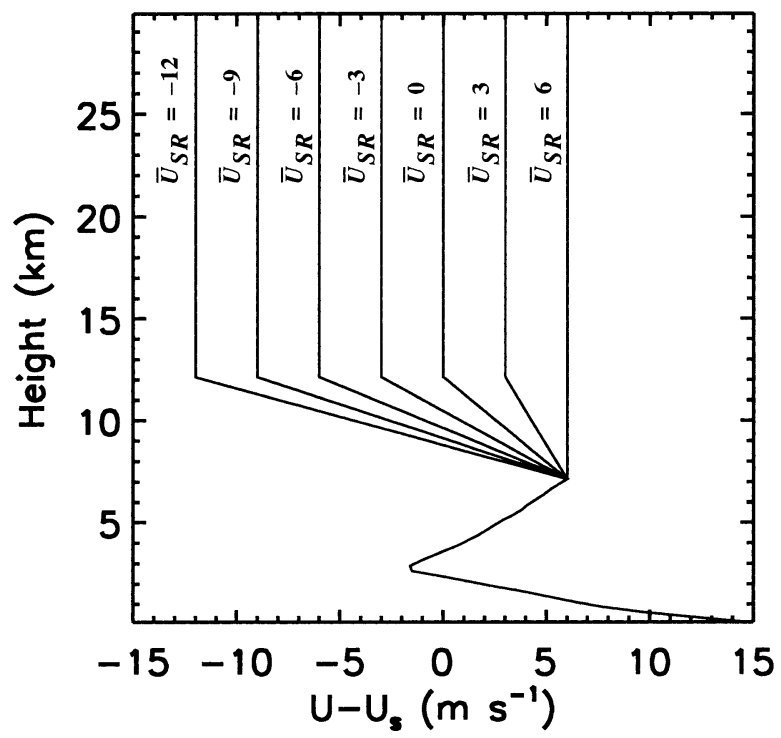

FIG. 4. Zonal wind profiles used to initialize the upper-level shear runs. Winds are plotted in meters per second, relative to the storm moving at an average speed $U_{s}=-16 \mathrm{~m} \mathrm{~s}^{-1}$. The storm-relative mean wind $\bar{U}_{\mathrm{SR}}$ is noted for each profile for reference.

averaged momentum flux $\bar{F}_{m}$. Figure 6 shows the eastward and westward $\bar{F}_{m}$ as a function of storm relative mean wind $\bar{U}_{\mathrm{SR}}$ for the regular upper-level shear runs. The different symbols in the figures correspond to momentum flux averages over the different storm stages. Each symbol in the lines corresponds to the domainaveraged flux from a simulation initialized with one of the wind profiles shown in Fig. 4.

For all periods of storm development, there is a general trend for the westward $\bar{F}_{m}$ to decrease, and for the eastward $\bar{F}_{m}$ to increase as the westward shear in the 7 to $12-\mathrm{km}$ layer increases, and $\bar{U}_{\mathrm{SR}}$ decreases. The change in westward $\bar{F}_{m}$ is much greater than the change in eastward $\bar{F}_{m}$ between the different upper-level shear simulations. Westward momentum flux is smaller by a factor of 6 in the case of strong upper-level shear $\left(\bar{U}_{\mathrm{SR}}=\right.$ $\left.-12 \mathrm{~m} \mathrm{~s}^{-1}\right)$ as compared to the case of no upper-level shear $\left(\bar{U}_{\mathrm{SR}}=6 \mathrm{~m} \mathrm{~s}^{-1}\right)$. On the other hand, on average eastward momentum flux is larger only by a factor of 0.5 in the case of strong upper-level shear as compared to the case of no upper-level shear.

Figure 6 also shows that gravity waves are not generated symmetrically in the east and west direction, nor is their momentum flux constant with time. Eastward momentum flux is greater than the westward momentum flux carried by the generated gravity waves in all the simulations but especially in simulations with strong upper-level westward shear. For the simulation with $U_{\mathrm{SR}}$ $=-12 \mathrm{~m} \mathrm{~s}^{-1}$, eastward momentum flux exceeds the westward momentum flux by a factor of 23 .

Both westward and eastward momentum flux carried by gravity waves are largest in the developing stage of the storm, corresponding to large values of CA. Al- 
a)

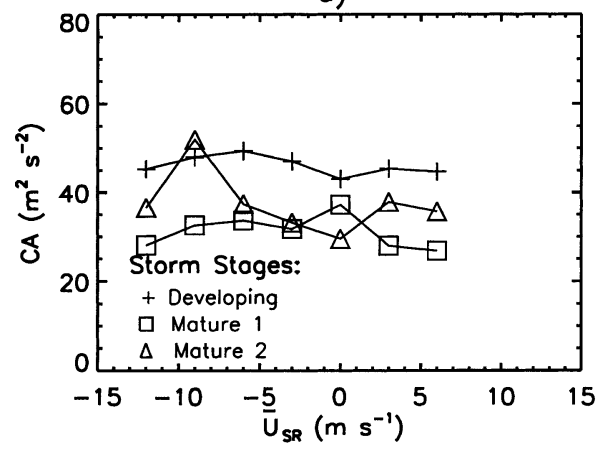

b)

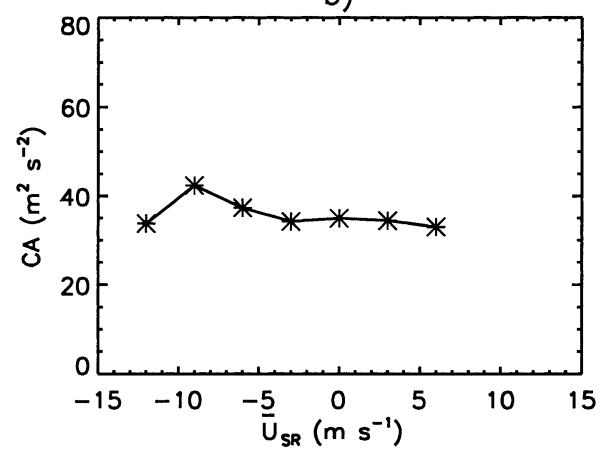

FIG. 5. Convective activity for upper-level shear runs averaged over three stages of storm development (a) separately and (b) together. Convective activity (in $\mathrm{m}^{2} \mathrm{~s}^{-2}$ ) is plotted vs the storm-relative mean wind $\bar{U}_{\mathrm{SR}}$ associated with each upper-level shear simulation. Each plotting symbol corresponds to a simulation initialized with one of the zonal wind profiles shown in Fig. 4.

though the convective activity values in the two mature stages are not always the same, the differences are too small to link them to the temporal evolution of momentum flux magnitudes.

In nearly all the upper-level shear simulations, eastward momentum flux decreases as the squall line develops. Westward momentum flux is largest in the developing stage, and is slightly larger in the mature stage 2 as compared to mature stage 1 . This temporal distribution of westward momentum flux is similar to that of the convective activity shown in Fig. 5, implying that the magnitude of westward momentum flux carried by gravity waves is strongly related to the strength of convection.

In all stages of storm evolution, the maximum value of stratospheric westward momentum flux is obtained when there is little or no shear above $7 \mathrm{~km}$. Therefore, the shear above that level must in some way act to reduce westward wave propagation. This also implies that most of the westward momentum flux is generated mainly below the level of $7 \mathrm{~km}$ and that the westward shear is preventing some of those waves from reaching the stratosphere. Since the squall line structure in the runs presented above is virtually the same, we propose that gravity waves generated by the heating mechanism are the same for these simulations. The structure of the waves that propagate into the stratosphere, is however, greatly modified by the upper-level shear. In order to test this theory, and to illuminate the wave generation and filtering mechanisms, we examine the momentum flux phase speed spectra for selected simulations.

\section{4) SPectral Characteristics}

The mechanisms responsible for changes in stratospheric wave momentum flux as a result of changes in upper-level tropospheric shear are best seen by comparing the phase speed spectrum for the case of no upper-level shear $\left(U_{\mathrm{SR}}=6 \mathrm{~m} \mathrm{~s}^{-1}\right)$ to the spectrum for the case with strongest shear $\left(U_{\mathrm{SR}}=-12 \mathrm{~m} \mathrm{~s}^{-1}\right)$. Figure 7 compares the phase speed spectra for those two simulations averaged over the different stages of storm development separately, as well as together. The spectra are shown for all these time periods to illustrate that the differences are not an artifact of the selected averaging intervals.

We suggest that the reduction of westward $\bar{F}_{m}$ by upper-level shear occurs through a mechanism similar to critical-level filtering as explained below. Linear theory predicts that waves cannot pass through a critical level, where the phase speed is equal to the background wind speed. The presence of scale-selective hyperdiffusion in the model assures that as a wave approaches a critical level it is damped and transfers momentum to the mean flow (Alexander and Holton 1997). Although the dynamics in the troposphere are nonlinear, this theory seems to be a good approximation for the wave filtering in the simulations: as shown in Fig. 7, in the stong upper-level shear simulation (solid line) the westward momentum flux is indeed zero between -12 and $0 \mathrm{~m} \mathrm{~s}^{-1}$, in the phase speed range in which waves would be filtered out if critical-level filtering was present.

The phase speed spectra in Fig. 7 also show how the eastward $\bar{F}_{m}$ is affected by westward shear. Increased westward shear primarily increases wave momentum flux at low phase speeds, between -12 and $15 \mathrm{~m} \mathrm{~s}^{-1}$, and shifts the eastward phase speed peak to a lower value by about $5 \mathrm{~m} \mathrm{~s}^{-1}$. This increase in eastward $\bar{F}_{m}$ resembles that caused by the obstacle effect presented by Pfister et al. (1993), where westward flow over the obstacle produces eastward-propagating waves with low phase speeds. In the squall line simulations with varying upper-level shear, latent heat release occurs mostly below the level of $7 \mathrm{~km}$, right below the upper-level shear zone, and the resulting displacement of the isentropes in this region can be thought of as of an obstacle. As predicted by the obstacle effect, increased upper-level shear in the region above the heating produces eastward- 
a) Westward Momentum Flux

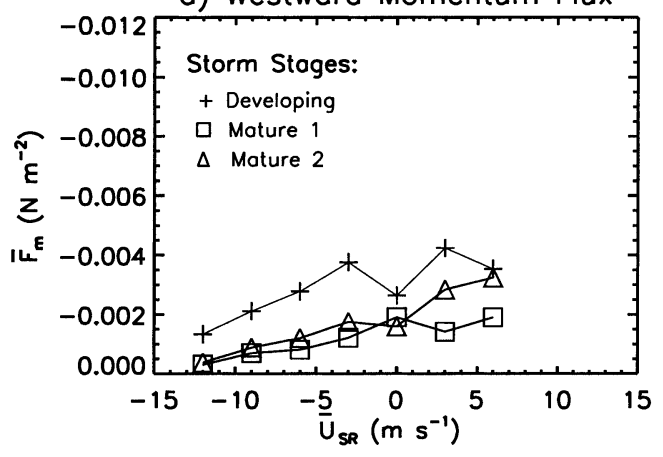

c) Westward Momentum Flux

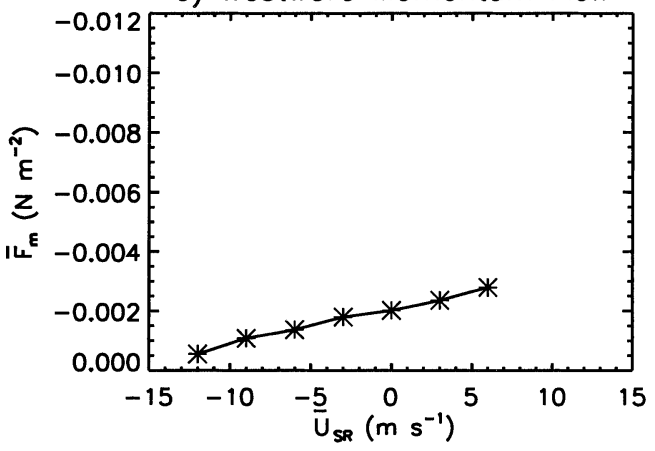

b) Eastward Momentum Flux

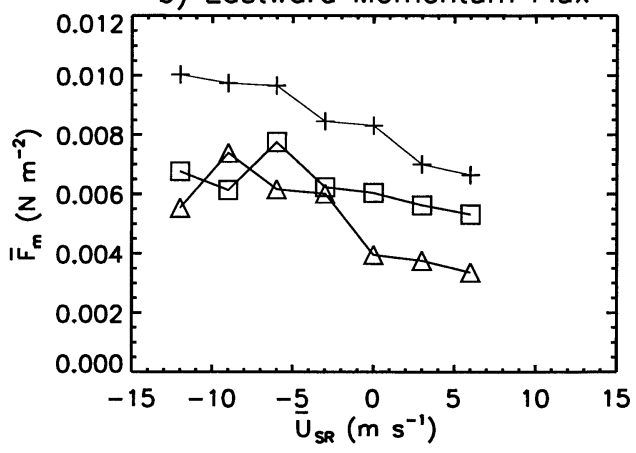

d) Eastward Momentum Flux

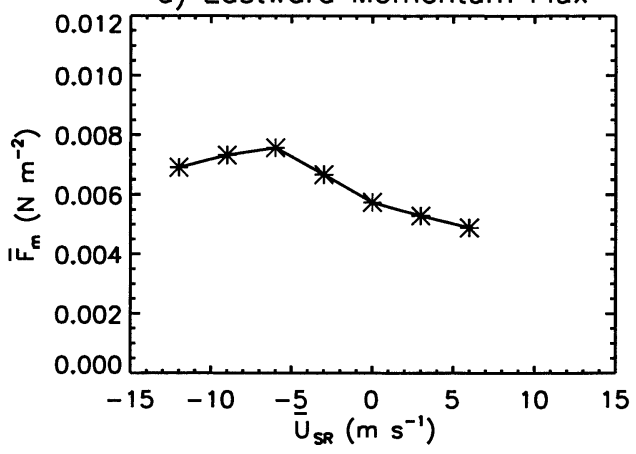

FIG. 6. Domain-averaged westward (left panels) and eastward (right panels) momentum fluxes (in $\mathrm{N} \mathrm{m}^{-2}$ ) vs the storm-relative mean wind $\bar{U}_{\mathrm{SR}}$ for all upper-level shear runs averaged over three stages of storm development separately (top panels) and together (bottom panels).

propagating waves with low phase speeds relative to the mean wind, as shown in Fig. 7. Note that, since the mean wind above the storm is $-12 \mathrm{~m} \mathrm{~s}^{-1}$ relative to the storm, waves with negative storm-relative phase speeds between -12 and $0 \mathrm{~m} \mathrm{~s}^{-1}$ carry positive momentum. The low phase speed gravity waves generated by the obstacle effect have distinctly different properties from the typical gravity waves generated by the storm's deep heating region. This can be illustrated by comparing the two-dimensional momentum flux spectra for the case of no shear to the case of strong shear shown in Fig. 8.

Figure 8 a shows that in the simulations with no shear above $7 \mathrm{~km}$, gravity waves are generated with a wide range of horizontal wavenumbers and frequencies, however nearly all the gravity waves have similar vertical wavelengths between 9 and $12 \mathrm{~km}$. Figure $8 \mathrm{~b}$ shows that similar gravity waves are present in the simulation with strong upper-level shear, however, there is also a substantial amount of wave momentum flux associated with gravity waves with vertical wavelengths less than $9 \mathrm{~km}$. These waves have frequencies less than $0.01 \mathrm{cy}-$ cles per minute and horizontal wavenumbers less than 0.0075 cycles per kilometer, corresponding to waves with periods greater than $100 \mathrm{~min}$, and horizontal wavelengths longer than $130 \mathrm{~km}$. These low-frequency, long horizontal wavelength gravity waves match quite well the characteristics of waves generated by the obstacle effect presented by Pfister et al. (1993).

It should be noted that even though the two simulations, one with no upper-level shear and one with strong upper-level shear, have almost the same convective activity, and same magnitude updrafts and rain rates, the time evolutions of vertical velocity at given points in the domain are different, and therefore gravity waves are excited with slightly different frequencies. Close comparison of Figs. 8a and $8 \mathrm{~b}$ shows that at frequencies greater than 0.01 cycles per minute, the momentum flux at a given frequency can be higher or lower in the simulation with strong upper-level shear as compared to the simulation with no upper-level shear. However, at frequencies less than 0.01 cycles per minute, the wave momentum flux is always greater in the simulation with strong upper-level shear. Therefore wave momentum flux integrated over frequencies greater than 0.01 cycles per minute is approximately the same in both simulations, whereas the momentum flux at frequencies less than 0.01 cycles per minute is much greater in the simulation with strong upper-level shear as compared to the simulation with no upper-level shear.

The results presented in this section suggest that all westward-propagating gravity waves are generated by the heating region below $7 \mathrm{~km}$. Some of these waves are then filtered out by the westward shear zone through 
a) Developing Stage

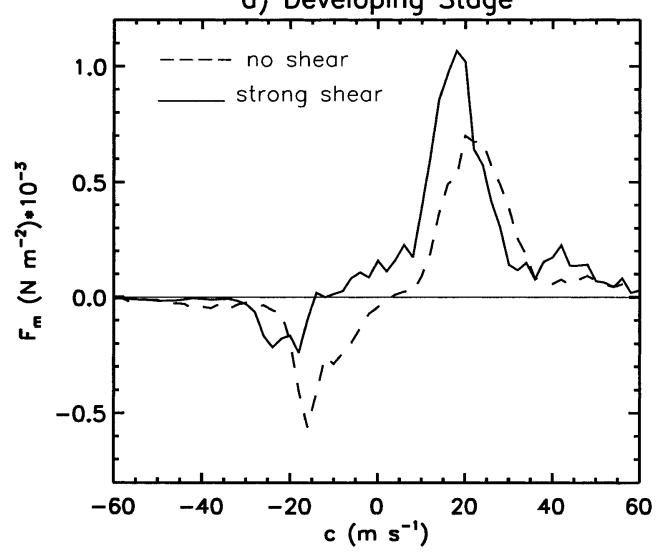

c) Mature Stage 2

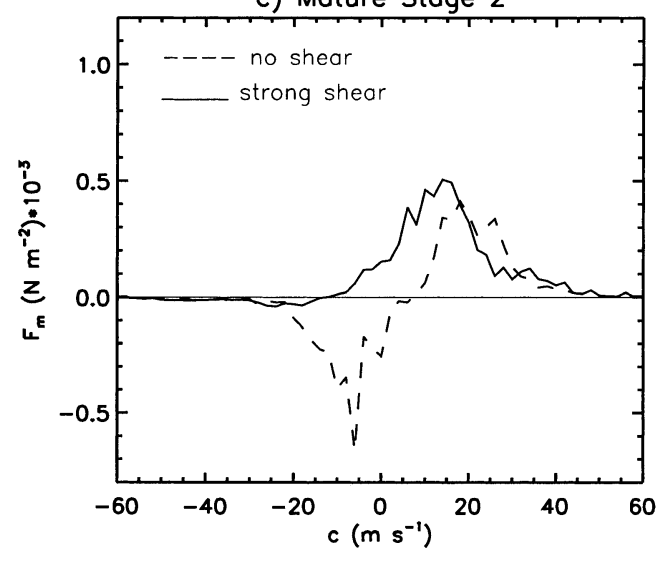

b) Mature Stage 1

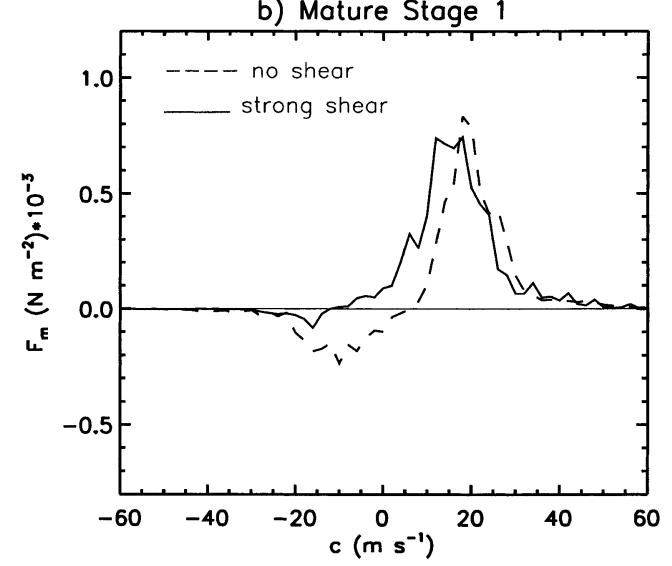

d) All Stages

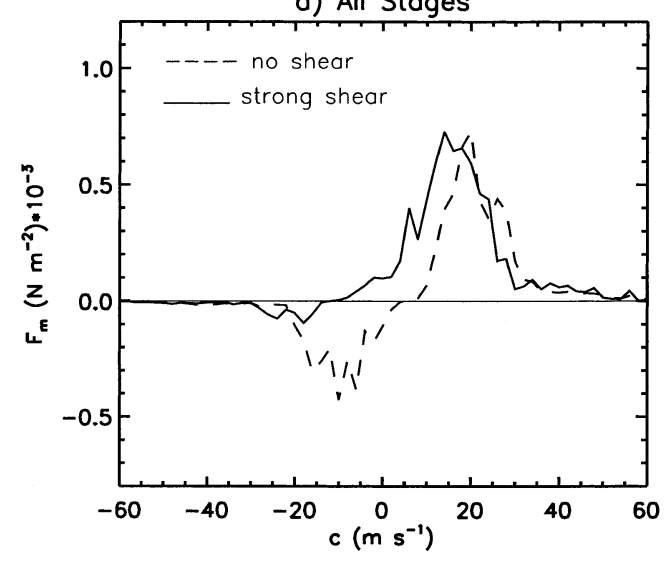

FIG. 7. Spectra of momentum flux vs phase speed $c$ in the frame of reference moving with the storm. Spectra are shown for the simulations with no upper-level shear $\left(U_{\mathrm{SR}}=6 \mathrm{~m} \mathrm{~s}^{-1}\right)$ in a dashed line, and for the simulation with strong upper-level shear $\left(U_{\mathrm{SR}}=-12 \mathrm{~m} \mathrm{~s}^{-1}\right)$ in a solid line. The spectra are averaged over (a) the developing stage, (b) mature stage 1, (c) mature stage 2, and (d) all three stages of storm development together. Initial zonal wind profiles corresponding to the phase speed spectra are shown in Fig. 4.

the filtering mechanism. Most of the eastward-propagating waves also originate in the heating region, however additional eastward propagating waves appear in the stratosphere in the presence of an upper-level westward shear zone.

\section{5) Generation MECHANiSMS VERSUS OTHER EFFECTS}

It was noted in the above section, that eastward gravity wave activity is enhanced in the presence of a westward shear zone. Although the additional waves generated in the presence of westward shear are very similar to those generated by the obstacle effect, results presented in the above section must be interpreted with care since zonal wind also acts to refract gravity waves and to alter their group velocities.

The vertical group velocity of internal gravity waves is defined as

$$
c_{g z}=\frac{\partial \omega}{\partial m}= \pm \frac{N k m}{\left(k^{2}+m^{2}\right)^{3 / 2}} .
$$

For hydrostatic waves this relationship can be rewritten as a function of the intrinsic zonal phase speed $\hat{c}$ :

$$
c_{g z}= \pm \frac{N k}{m^{2}}= \pm \frac{\hat{\omega}}{m}= \pm \frac{\hat{\omega} \hat{c}}{N},
$$

or in terms of phase speed $c$ relative to the frame of reference moving with the storm, and storm-relative mean wind $\bar{U}_{\mathrm{SR}}$ :

$$
c_{g z}= \pm \frac{\left(c-\bar{U}_{\mathrm{SR}}\right)^{2} k}{N} .
$$

The above imply that waves propagating eastward relative to the storm, will have higher vertical group velocities in the presence of westward storm-relative mean wind as compared to a situation with zero or eastward storm-relative mean wind. Gravity wave group veloci- 


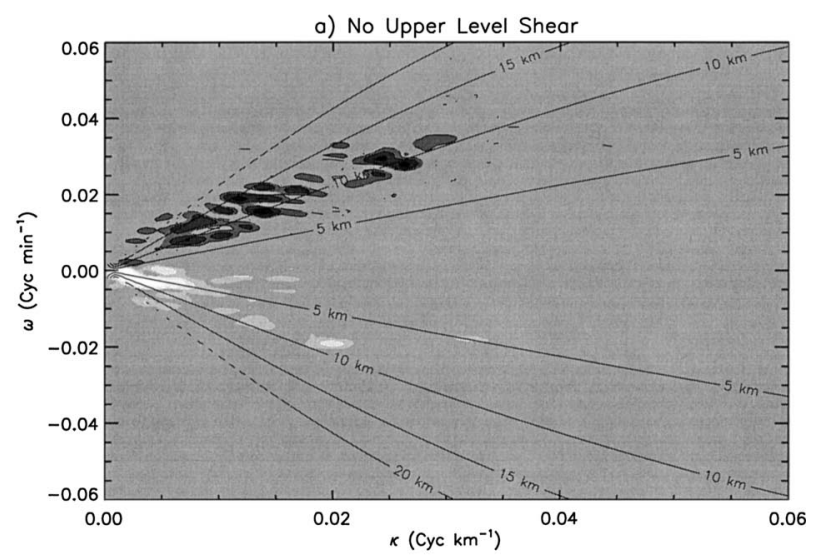

b) Strong Upper Level Shear

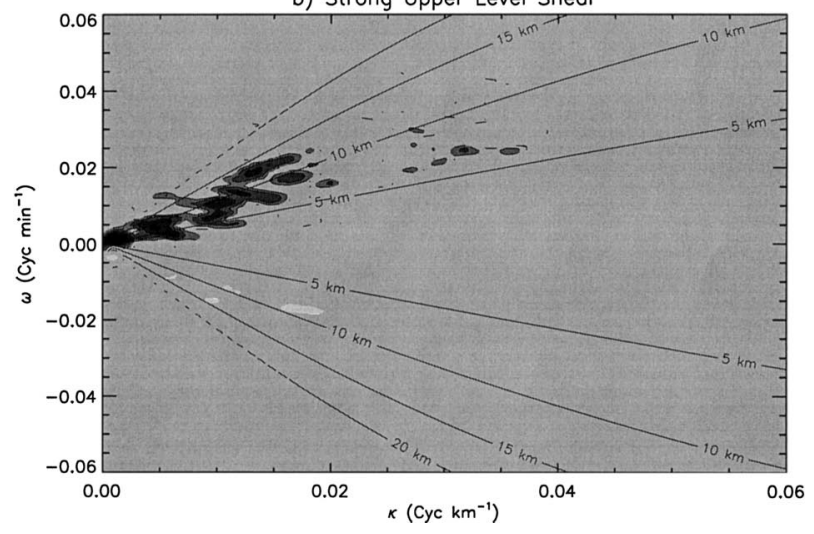

FIG. 8. Momentum flux (in $\mathrm{N} \mathrm{m}^{-2}$ ) as a function of frequency $\omega$ (in cycles per minute), and horizontal wavenumber $k$ (in cycles per kilometer). Positive values are shaded in dark, whereas negative values are in light. Contour and shading intervals are $(-24,-12,-6$, $-3,-1,1,3,6,12,24) \times 10^{-5}$. (Values between -1 and 1 are shaded in light gray.)

ties and therefore propagation times from wave source to tropopause are affected mostly for waves with low storm-relative phase speeds $c \leq 10 \mathrm{~m} \mathrm{~s}^{-1}$, and low frequencies $\omega \leq 0.005$ cycles per minute. For example, a gravity wave with $k=0.005$ cycles per kilometer, $\omega$ $=0.003$ cycles per minute, and $c=10 \mathrm{~m} \mathrm{~s}^{-1}$, has a vertical group velocity of $\sim 4.6 \mathrm{~km} \mathrm{~h}^{-1}$ when $\bar{U}_{\mathrm{SR}}=$ $-12 \mathrm{~m} \mathrm{~s}^{-1}$, and a group velocity of only $0.15 \mathrm{~km} \mathrm{~h}^{-1}$ when $\bar{U}_{\mathrm{SR}}=6 \mathrm{~m} \mathrm{~s}^{-1}$. Therefore when considering differences in the wave spectrum between the simulation with strong upper-level shear $\left(\bar{U}_{\mathrm{SR}}=-12 \mathrm{~m} \mathrm{~s}^{-1}\right)$ and no upper-level shear $\left(\bar{U}_{\mathrm{SR}}=6 \mathrm{~m} \mathrm{~s}^{-1}\right)$, it is important to realize that even if some waves with low frequencies and low eastward phase speeds were generated in the simulation with no upper-level shear, they would have had too-small group velocities to propagate to the analysis level during the chosen analysis periods. We will refer to these waves as "uncertain" because they could have been generated in the simulation with no upperlevel shear, but just did not have enough time to propagate to the analysis level.
For the mature stage 1 of the strong upper-level shear simulations, the magnitude of momentum flux carried by these uncertain waves was assessed by first calculating an approximate propagation time to the tropopause, based on $\bar{U}_{\mathrm{SR}}=6 \mathrm{~m} \mathrm{~s} \underline{\mathrm{s}}^{-1}$, of all gravity waves generated in that simulation $\left(\bar{U}_{\mathrm{SR}}=-12 \mathrm{~m} \mathrm{~s}^{-1}\right)$. Subsequently their propagation time to the tropopause was calculated based on the source level of $7 \mathrm{~km}$. This calculation yields an overestimate of the propagation time to tropopause of waves generated above $7 \mathrm{~km}$, and therefore is an absolute upper limit and likely to be an overestimate of the momentum flux carried by the uncertain gravity waves. In the mature stage 1 of the strong upperlevel shear simulation, the momentum flux carried by uncertain waves was $25 \%$ of the total eastward momentum flux carried by gravity waves. In other words, $25 \%$ of the total eastward momentum flux in the strong upper-level shear simulations was carried by gravity waves that could have been generated in the simulation with no upper-level shear, but had not enough time to propagate to the tropopause. Sixty-six percent of these waves in the strong upper-level shear simulations had phase speeds less than $10 \mathrm{~m} \mathrm{~s}^{-1}$.

Therefore, in terms of the gravity wave generation mechanisms, phase speed spectra presented in Fig. 7 may be overestimating the effect of wind shear primarily for waves with phase speeds less than $10 \mathrm{~m} \mathrm{~s}^{-1}$. However, the presented spectra are a fairly accurate representation of the gravity wave spectrum actually reaching the tropopause. This is because waves that are refracted by the westward shear zone to lower vertical wavelengths, and which have low group velocities, are also most likely to suffer dissipation. In the model used in this study, the wave dissipation processes are parameterized using fourth order dissipation. This dissipation may mimic dissipation processes in the real atmosphere and may be another reason why waves with low frequencies and phase speeds are not present in simulations with eastward storm-relative mean wind.

In summary, the phase speed spectra presented in Fig. 7 , fairly accurately display the changes in gravity wave activity that result from changes in tropospheric wind shear; however, a portion of the differences may be due to changes in wave group velocity and dissipation, and not due to changes in wave-generation mechanisms.

\section{d. Varying middle- and lower-level shear}

In the previous section it was shown how upper-level shear modifies the structure of gravity waves generated by the heating below the level of $7 \mathrm{~km}$. The waves generated below that level however did not appear to be generated isotropically. Figures 7 and 8 a show that in the case of no upper-level shear there are big differences between waves propagating in the eastward and westward directions. In this section we will examine the role of low- and midlevel shear in shaping the gravity 


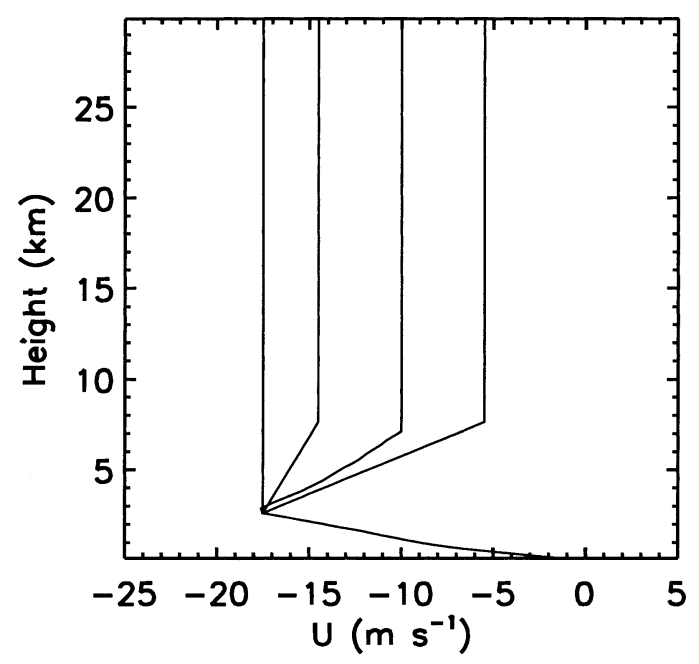

FIG. 9. Zonal wind profiles used to initialize runs with varying midlevel shear (for strong jet runs). Winds are plotted in meters per second, relative to the ground.

wave spectrum. There is no shear above $7 \mathrm{~km}$ in these simulations.

\section{1) EXPERIMENTAL DESIGN}

The initial wind profile is altered in the middle level of the troposphere, between 2.5 and $7 \mathrm{~km}$, while keeping the low-level shear the same as in the basic case at 6.4 $\times 10^{-3} \mathrm{~s}^{-1}$. The strength of the shear in the midlevel is varied from 0 to $4.8 \times 10^{-3} \mathrm{~s}^{-1}$ in four simulations. The wind profile in which the wind speed increases by $16 \mathrm{~m} \mathrm{~s}^{-1}$ in the lowest $2.5 \mathrm{~km}$ of the troposphere observed in the basic case is referred to here as the strong low-level jet. The simulations in which the wind profile has a strong low-level jet and the midlevel shear varies are referred to as the strong jet runs. The initial wind profiles for these simulations are shown in Fig. 9, plotted relative to the ground.

Second, few simulations were performed with varying midlevel shear with a weak low-level shear of 4.4 $\mathrm{s}^{-1}$. Since the differences between these simulations were similar to the differences between the strong jet runs, the details of the simulations are not presented in this paper. It is worth mentioning however, that the simulations with weak and strong low-level shear had virtually the same momentum flux values for the same values of storm-relative mean wind.

\section{2) Storm Structure}

Figure 10 shows the convective activity for the midlevel shear simulations. Unlike the upper-level shear, midlevel shear changes the storm's convective activity considerably. There is a 50\% decrease in CA in the case of strong midlevel shear as compared to the case with no midlevel shear.

\section{3) Momentum flux Magnitudes}

Figure 11 compares the distribution of momentum flux for the strong jet runs averaged over the different stages of storm development. As for upper-level shear runs, momentum flux is plotted as a function of stormrelative mean wind $\bar{U}_{\mathrm{SR}}$ defined by (4). Westward momentum is fairly constant in the simulations with nonzero midlevel shear. However, there is about a $50 \%$ decrease in the magnitude of the westward momentum flux in these simulations as compared to the simulation with only low-level shear. This decrease is similar to the decrease in the storm's convective activity (Fig. 10), implying that midlevel shear does not strongly affect the mechanisms that generate westward-propagating gravity waves.

On the other hand, Figs. $11 \mathrm{~b}$ and $11 \mathrm{~d}$ show a more pronounced dependence of eastward $\bar{F}_{m}$ on $\bar{U}_{\mathrm{SR}}$. Eastward $\bar{F}_{m}$ decreases with increasing eastward shear in the middle level, or increasing $\bar{U}_{\mathrm{SR}}$. In all stages of storm development, eastward $\bar{F}_{m}$ decreases by at least $200 \%$ in the strong midlevel shear case as compared to the no midlevel shear case. This decrease is much greater than the decrease in westward $\bar{F}_{m}$, and cannot be explained by the decrease in the storm's convective activity. The possible mechanism responsible for the additional de- a)

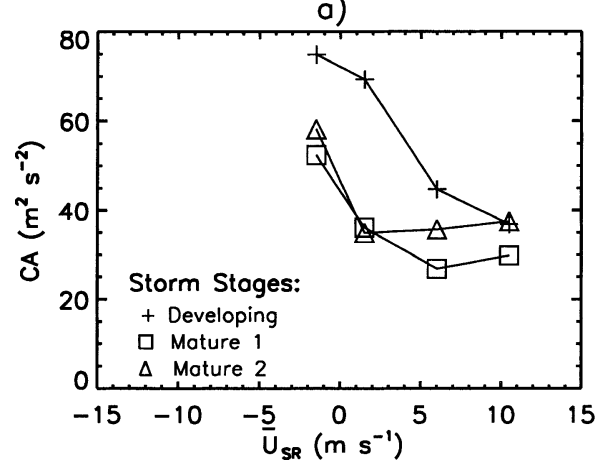

b)

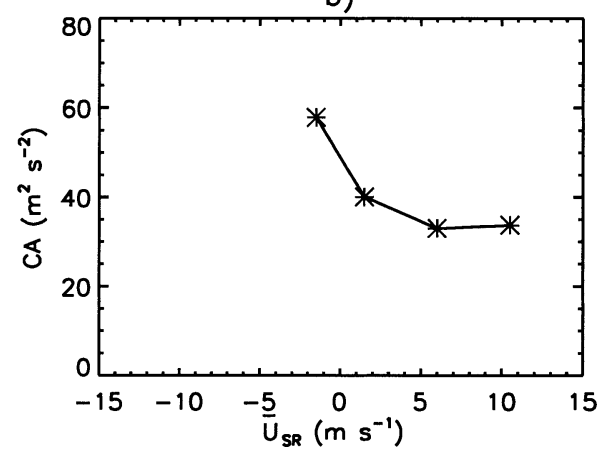

FIG. 10. Same as in Fig. 5, but for simulations with varying midlevel shear. 
a) Westward Momentum Flux

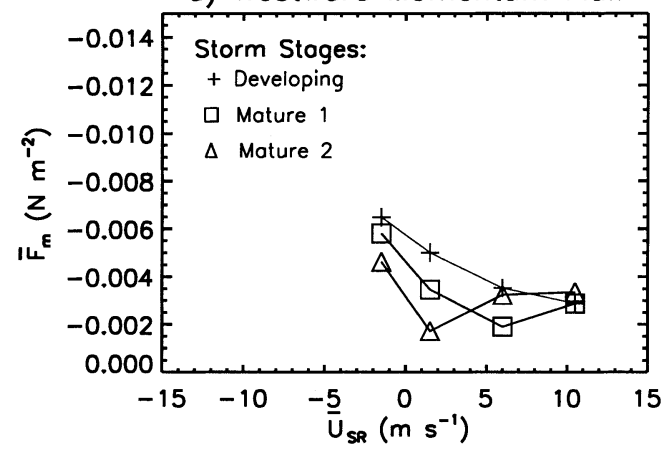

c) Westward Momentum Flux

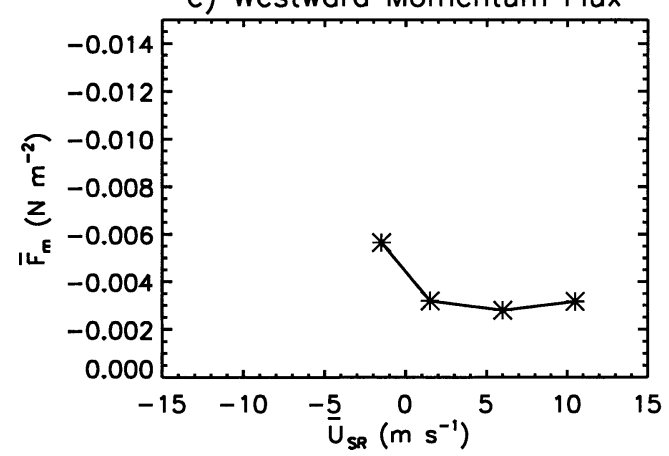

b) Eastward Momentum Flux

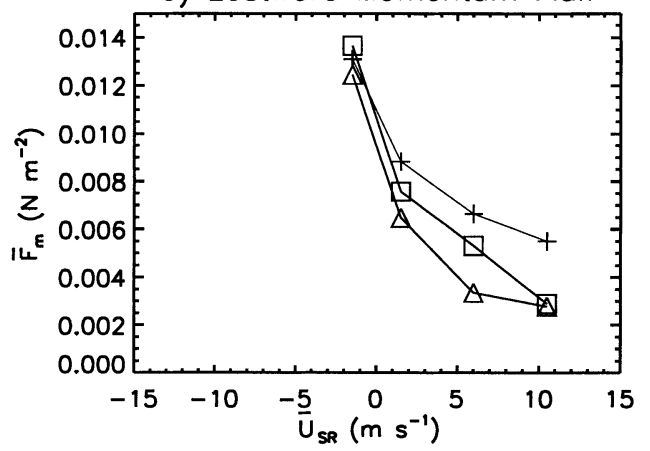

d) Eastward Momentum Flux

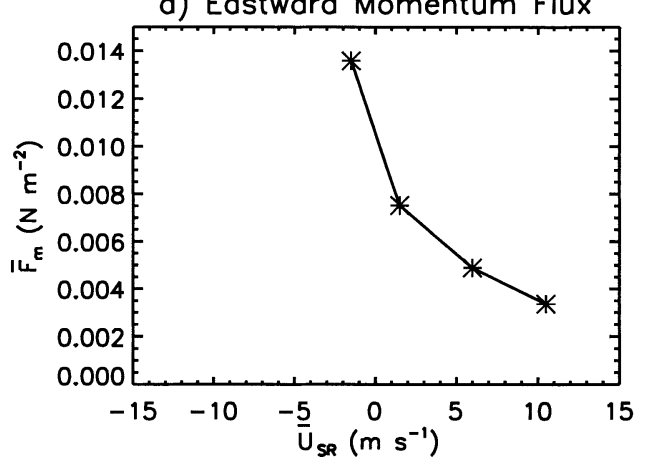

FIG. 11. Same as in Fig. 6, but for simulations with varying midlevel shear.

crease in eastward momentum flux will be explored by looking at the $\bar{F}_{m}$ phase speed spectra.

\section{4) Phase SPEEd SPECTRA}

Momentum flux phase speed spectra averaged over mature stage 1 for the case of no midlevel shear $\bar{U}_{\mathrm{SR}}$ $\left.=-1.5 \mathrm{~m} \mathrm{~s}^{-1}\right)$ and the case of strong midlevel shear $\left(\bar{U}_{\mathrm{SR}}=10.5 \mathrm{~m} \mathrm{~s}^{-1}\right)$ are shown in Fig. 12. Spectra are only shown for the mature stage 1 since simlar characteristics are observed in the other storm stages.

Figure 12 shows that in the presence of midlevel tropospheric shear, maximum amplitude westward momentum flux values are associated with waves with lower, less negative phase speeds as compared to the no midlevel shear case. However, the maximum westward momentum flux amplitudes are associated with the same phase speeds in the frame of reference moving with the wind speed at the $5.5-\mathrm{km}$ level. To demonstrate this effect, we will focus on the westward momentum flux distribution in Fig. 12. In the case of strong midlevel shear, the leftmost peak in westward momentum flux occurs at $-14 \mathrm{~m} \mathrm{~s}^{-1}$ as compared to at $-22 \mathrm{~m} \mathrm{~s}^{-1}$ in the case with no midlevel shear. However, relative to the wind near $5.5 \mathrm{~km}$, close to the top of the heating region, the maximum westward momentum flux occurs in both simulations at the phase speed of $\sim 20 \mathrm{~m} \mathrm{~s}^{-1}$. This is an approximate value, since the phase speed spectrum resolution is only $2 \mathrm{~m} \mathrm{~s}^{-1}$. Generation of gravity waves with maximum amplitude momentum flux at the intrinsic phase speed of approximately $20 \mathrm{~m} \mathrm{~s}^{-1}$ can also be noted for the eastward propagating waves, however other effects are also present. In summary, these mid-level shear experiments suggest that the convection generates a gravity wave spectrum that peaks at \pm 20 $\mathrm{m} \mathrm{s}^{-1}$ phase speed in the frame of reference moving with the wind at $5.5-\mathrm{km}$ altitude. The reason gravity waves are generated at the particular phase speed of $\sim 20 \mathrm{~m} \mathrm{~s}^{-1}$ is discussed in section 4 .

Although there is symmetry in the phase speeds of waves carrying maximum eastward and westward momentum flux that originate near the $5.5-\mathrm{km}$ level, midlevel shear affects the momentum fluxes of these waves differently. The domain-averaged momentum flux for westward-propagating waves is relatively constant throughout the midlevel shear simulations. Phase speed spectra show no evidence of wave generation analogous to the obstacle effect produced by the upper-level shear. This could be due to the fact that in varying midlevel shear simulations the shear region is well within the heating layer, whereas, in the upper-level shear runs, the shear layers were located above the heating. Thus, when shear is confined in the region below $7 \mathrm{~km}$, the background flow is going through rather than over the heating, not creating an obstacle effect.

The domain-averaged eastward momentum flux de- 

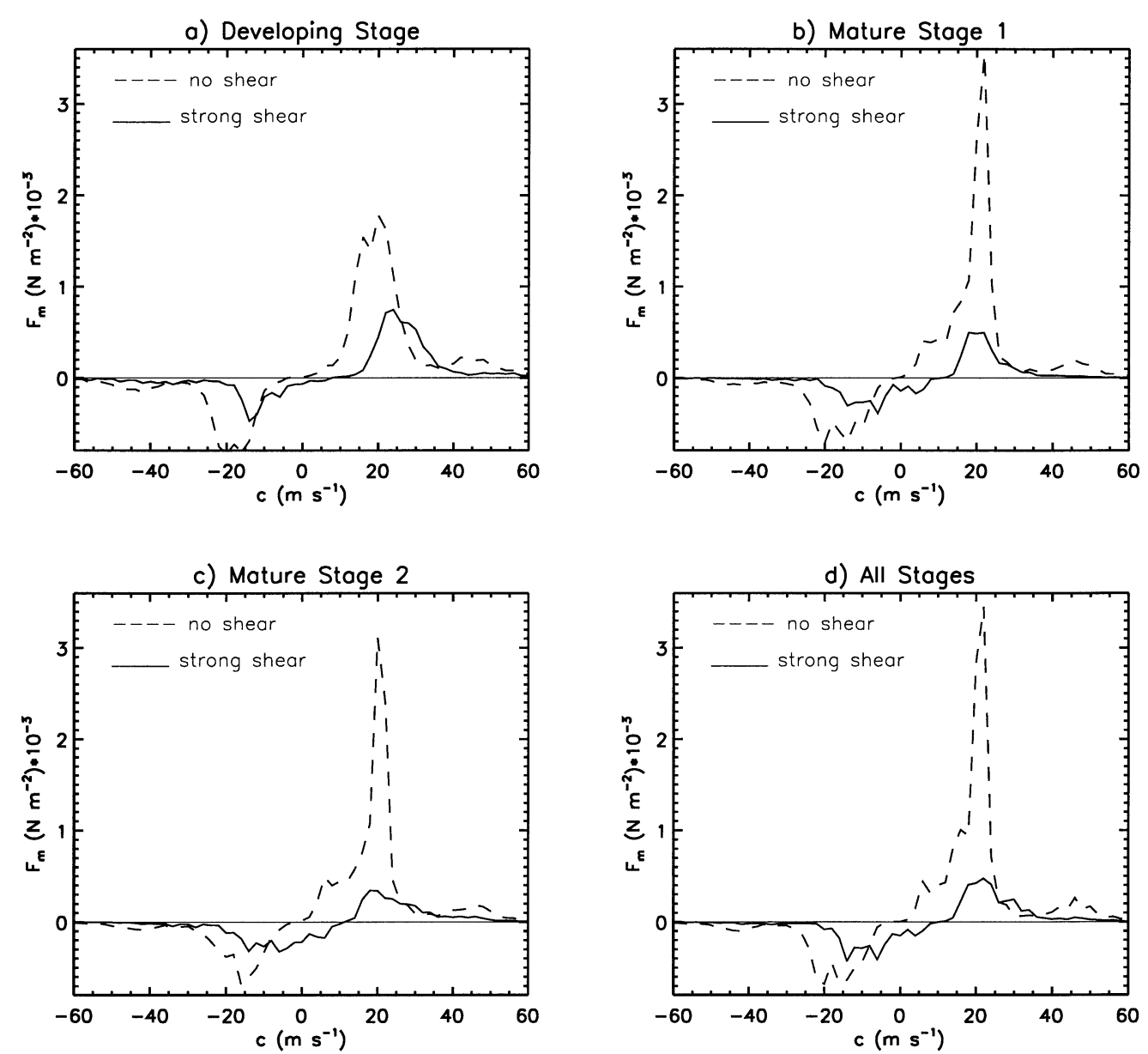

FIG. 12. Same as in Fig. 7, but for the simulation with no midlevel shear $U_{\mathrm{SR}}=-1.5 \mathrm{~m} \mathrm{~s}^{-1}$ (dashed line), and the simulation with strong midlevel shear $U_{\mathrm{SR}}=10.5 \mathrm{~m} \mathrm{~s}^{-1}$ (solid line).

creases with increased midlevel eastward shear. Eastward momentum flux appears to be reduced through the filtering mechanism. The region of phase speeds in which eastward momentum flux should be zero according to linear theory of critical-level filtering is between 0 and $10.5 \mathrm{~m} \mathrm{~s}^{-1}$ in the case of strong midlevel shear. Indeed, the phase speed spectrum for this simulation (solid line in Fig. 12) shows no eastward $F_{m}$ in that region, suggesting that waves with phase speeds equal to the wind speed relative to the storm at any level above $2.5 \mathrm{~km}$, cannot propagate upward into the stratosphere.

\section{5) Vertical waVelength SPECTRA}

In the above section it was found that eastward- and westward-propagating gravity waves are generated with a dominant phase speed of $20 \mathrm{~m} \mathrm{~s}^{-1}$ relative to the 5.5$\mathrm{km}$ level wind, and their structure is further altered by the midlevel shear. Below we demonstrate that the dominant intrinsic wave phase speed of $20 \mathrm{~m} \mathrm{~s}^{-1}$ at the generation level is determined by the vertical extent of the heating region.
The linear gravity wave dispersion relationship in the Boussinesq approximation can be expressed as

$$
\hat{\omega}^{2}=\frac{N^{2} k^{2}}{\left(k^{2}+m^{2}\right)},
$$

where $\hat{\omega}$ represents the intrinsic wave frequency; $k$ and $m$ are the horizontal and vertical wavenumbers, respectively; and $N$ is the buoyancy frequency. Assuming that the vertical wavelength $\lambda_{v}$ is much smaller than the horizontal wavelength $\lambda_{h}, m \gg k$, the intrinsic wave phase speed $\hat{c}$ can be written as

$$
\hat{c}=\frac{\hat{\omega}}{k}=\frac{N}{m}=\frac{N \lambda_{v}}{2 \pi} .
$$

Thus the intrinsic phase speed is proportional to the vertical wavelength of the wave.

Figure 13 shows the vertical wavelength spectra for the simulation with no midlevel shear for the mature stage 1. Both the eastward and westward spectra show the dominance of waves with the vertical wavelength of $5.5-6 \mathrm{~km}$. The spectra also show a peak near $4 \mathrm{~km}$, 


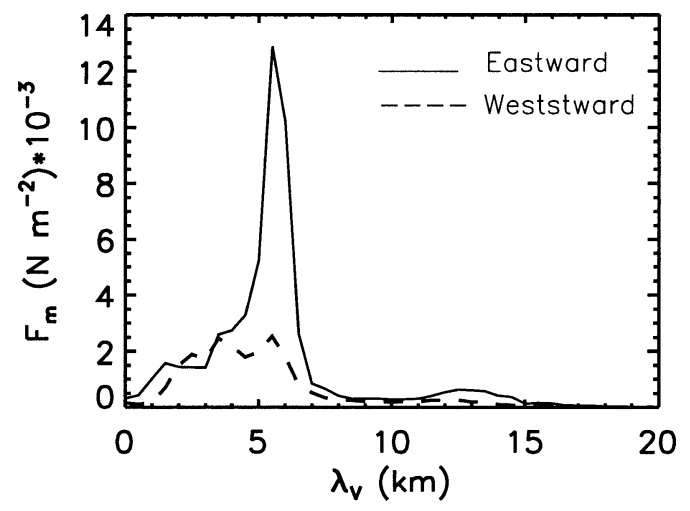

FIG. 13. Spectra of eastward (solid) and westward (dashed) momentum flux vs vertical wavelength $\lambda_{V}(\mathrm{~km})$ for the squall line simulation with only low-level shear. Wind profile used to initialize this simulation is shown in Fig. 9.

which is more pronounced for the westward propagating waves. By (10), the intrinsic phase speed corresponding to a 5.5-6-km vertical wavelength and stratospheric buoyancy frequency of $0.022 \mathrm{~s}^{-1}$ is $\approx 20 \mathrm{~m} \mathrm{~s}^{-1}$ corresponding well to the intrinsic phase speed of waves at the 5.5-km level shown in Fig. 12.

The dominant vertical wavelength range of about 5.5$6 \mathrm{~km}$ of the simulated stratospheric waves is approximately equal to the vertical extent of the tropospheric heating. In the squall line simulations, the dominant latent heating region extends to $\sim 6 \mathrm{~km}$. Therefore, the vertical wavelength spectra suggest that the deep heating wave-generating mechanism determines the approximate vertical wavelength of waves generated by convection. By (10) the vertical extent of the tropospheric heating also prescribes the intrinsic phase speed of gravity waves at their generation level.

The origin of the secondary peak at vertical wavelength of $\sim 4 \mathrm{~km}$ in Fig. 13, corresponding to the intrinsic phase speed of $14 \mathrm{~m} \mathrm{~s}^{-1}$, is not predicted by the deep heating mechanism. The following section will explore gravity wave generation by a simplified thermal forcing, and will attempt to determine whether the heating region could be responsible for prescribing two characteristic vertical wavelengths.

\section{Specified heating simulations}

In the previous section it was proposed that characteristics of gravity waves are primarily determined by the latent heating region and are modified by the horizontal wind profile. In order to verify the above speculations about how shear interacts with a thermal forcing, the convective storm was approximated by an oval shaped heating-cooling region with a specified monochromatic oscillation frequency. A fixed frequency source was used in these simulations to reduce the number of unknown parameters in the problem and to the facilitate the interpretation of the changes in gravity wave structure to vertical wind shear.

\section{a. Initial conditions}

All simulations are carried out in an atmosphere with a constant buoyancy frequency equal to $0.012 \mathrm{~s}^{-1}$, representative of the troposphere. Inclusion of a stable stratospheric layer was not necessary since the increased buoyancy frequency in the stratosphere only acts to refract the generated waves and gives no further insight into the wave-generation mechanisms. All of the wave generation for thermally forced waves occurs in the close vicinity of the heating region; gravity wave phase lines are clearly defined even right above the heating region. The vertical extent of the model domain for these simulations is shortened to $26 \mathrm{~km}$ to reduce computational time. The heating-cooling region is Gaussianshaped in the horizontal and has a half-sine wave structure in the vertical centered on $z=3 \mathrm{~km}$ with a quarter wavelength equal to $2 \mathrm{~km}$. The forcing function has the following form:

$$
Q(x, z, t)= \begin{cases}Q_{o} \exp \frac{-\left(x-x_{c}\right)^{2}}{x_{\mathrm{rad}}^{2}} \sin \frac{\pi\left(z-z_{c}+z_{\mathrm{rad}}\right)}{2 z_{\mathrm{rad}}} \sin \frac{2 \pi t}{T} & \text { for } 1125 \leq z \leq 5125 \mathrm{~m} \\ 0 & \text { for } z<1125 \mathrm{~m} \text { and } z>5125 \mathrm{~m},\end{cases}
$$

where, $x_{\mathrm{rad}}=3000 \mathrm{~m}, x_{c}=600 \mathrm{~km}, z_{c}=3125 \mathrm{~m}, z_{\mathrm{rad}}$ $=2000 \mathrm{~m}$, and $T=6000 \mathrm{~s}(100 \mathrm{~min})$.

As was noted in Fig. 8, the gravity waves generated in the squall line simulations all have similar vertical wavelengths, but have a broad range of horizontal wavenumbers and frequencies. The value of the period $T$ chosen in the specified heating simulations corresponds to one of the dominant wave frequencies present in the squall line simulations, however it is not the only wave frequency present. The specified heating experiments described in this section were repeated at other frequencies, however the main results were not altered.

The specified heating simulations were carried out for $66000 \mathrm{~s}$, or over $18 \mathrm{~h}$. Wave activity analyses shown below are at the level of $10 \mathrm{~km}$, during the last six cycles of the simulations, between 30000 and $66000 \mathrm{~s}$. Analyses were performed at different heights and during dif- 


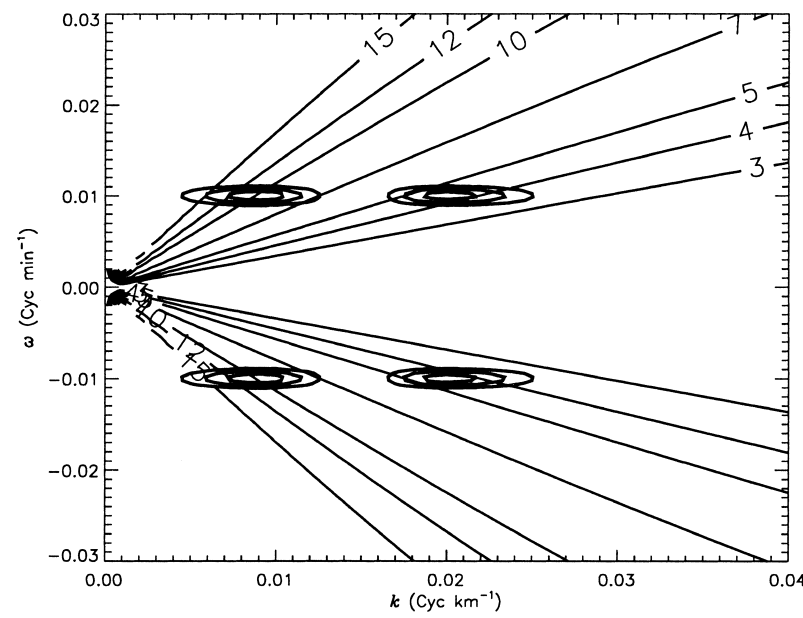

FIG. 14. Two-dimensional spectrum of momentum flux in horizontal wavenumber $k$ and frequency $\omega$ for the control simulation using specified heating. Thick lines are contours of momentum flux at 0.1 $\times 10^{-5} \mathrm{~N} \mathrm{~m}^{-2}$ intervals. The superimposed thin lines are contours of vertical wavelength calculated using the dispersion relation given by Eq. (9).

ferent time intervals but the main results of the study were not sensitive to these changes. The small differences that may arise when using different averaging intervals and analysis levels will be discussed further in the following sections.

\section{b. Control simulation}

The control simulation is carried out in a motionless atmosphere. A two-dimensional momentum flux spectrum averaged over the last six cycles of the control simulation is shown in Fig. 14. Overlaid are lines of constant vertical wavelength calculated from the dispersion relation (9). The generated wave field is symmetric in the east-west direction, and as expected, the dominant frequency of the waves is equal to the frequency of the thermal forcing of 0.01 cycles per minute. The spectrum shows two peaks in horizontal wavenumbers, at 0.008 and 0.02 cycles per kilometer, which correspond to the vertical wavelengths of about 10 and 4.5 $\mathrm{km}$ for waves with the dominant frequency. The $10-\mathrm{km}$ vertical wavelength is consistent with the deep heating mechanism, being approximately equal to twice the vertical extent of the prescribed heating region. The peak at the $4.5-\mathrm{km}$ vertical wavelength suggests that a thermal forcing region of specified depth can generate gravity waves with more than one dominant vertical wavelength. This occurs when the thermal heating region is elevated above the ground. A thermal heating region of similar form as in (11), but extending from the ground to the $5-\mathrm{km}$ level, generates gravity waves with only one dominant vertical wavelength of $10 \mathrm{~km}$ (not shown). The lifted heating region generated waves with two dominant vertical wavelengths, because more than one Fourier wave component is necessary to represent the shape of the heating profile in the region between 0 and $5 \mathrm{~km}$. In other words, the shape of the heating projects strongly on more than one Fourier mode.

The lifted heating region could explain the secondary peak at the vertical wavelength of $4-5 \mathrm{~km}$ in the spectra for the squall line simulation shown in Fig. 13. The latent heating region in the simulated squall line extends on average to the height of $6 \mathrm{~km}$, however, the individual convective cells are only about 3 to $4-\mathrm{km}$ tall and slowly propagate upward from the ground.

The range of dominant horizontal wavenumbers simulated in the control run is prescribed by the dispersion relation. Since the frequency of the waves is determined by the frequency of the heating-cooling region, and the vertical wavelength is determined by the vertical extent of the heating, the dominant horizontal wavenumbers must have values so as to satisfy the dispersion relation. Simulations were performed with altered horizontal scale of the heating region, however for the chosen frequency and vertical scale, these changes had no effect on the structure of the generated waves.

\section{c. Upper-level shear}

To examine how upper-level shear interacts with a thermal forcing, a specified heating simulation is performed with a $15 \mathrm{~m} \mathrm{~s}^{-1}$ westward shear through the top of the heating region: the shear layer starts at $3 \mathrm{~km}$, in the center of the heating, and continues to the height of $8 \mathrm{~km}$, going through and partially over the heating region. Figure $15 \mathrm{~b}$ shows the initial wind profile, and Fig. $15 \mathrm{a}$ shows the corresponding momentum flux phase speed spectrum for this simulation, and compares it to the phase speed spectrum for the control case with zero mean wind.

In the specified heating simulation with westward shear through the top of the heating region, $\bar{F}_{m}$ is reduced by critical-level filtering. Waves with westward $F_{m}$ and phase speeds between 0 and $-15 \mathrm{~m} \mathrm{~s}^{-1}$ can not propagate through the shear zone and are dissipated. The momentum flux spectra in Fig. 15a show a considerable decrease in momentum flux in that region as compared to the control case.

Figure 15a also shows that eastward wave propagation is enhanced by the shear zone near the top and just above the heating region. Firstly, this westward shear zone enhances momentum flux of eastward-propagating gravity waves with low positive phase speeds, $\sim 8 \mathrm{~m}$ $\mathrm{s}^{-1}$. The two-dimensional momentum flux spectrum (not shown) shows that these waves have the frequency of the forcing, but shorter horizontal scale than the waves in the control simulation. These waves are not present in the control simulation, since in the absence of westward shear, they have smaller vertical group velocities and are more affected by dissipation. Second, the westward shear zone generates weak waves with very low, almost zero, frequencies, and long horizontal and short vertical wavelengths. These waves correspond to the 

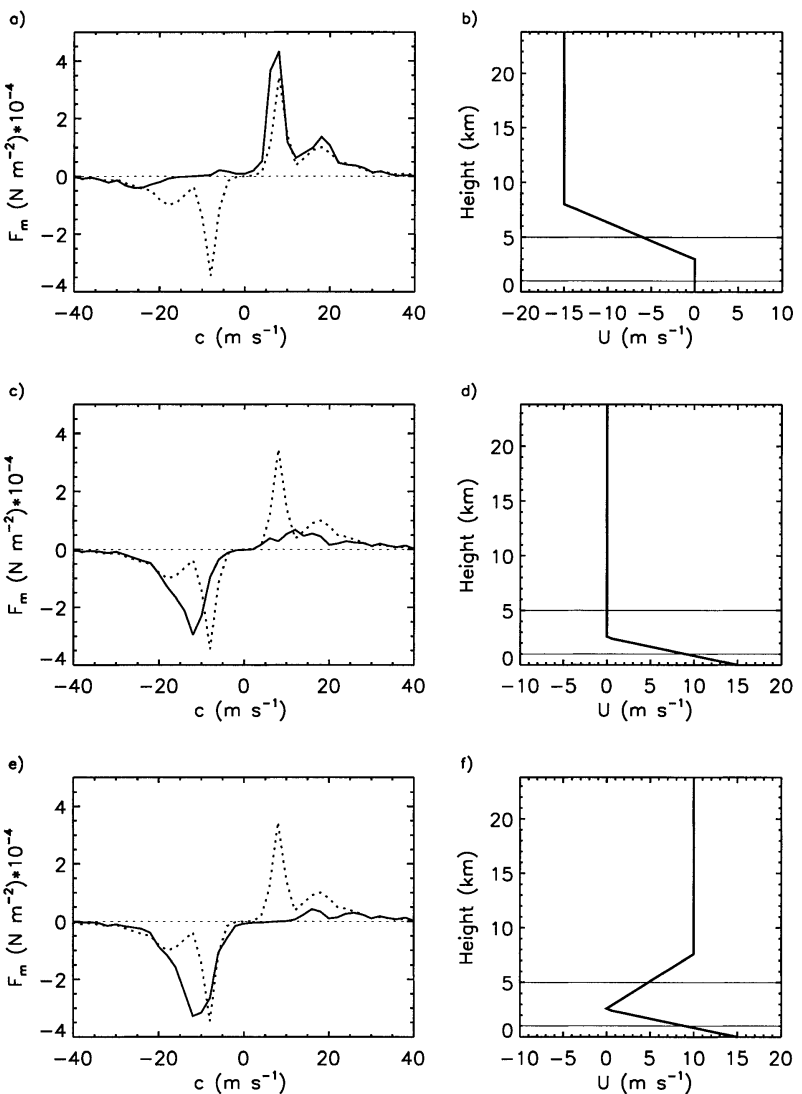

FIG. 15. Spectra of momentum flux vs phase speed $c$ relative to a stationary frame of reference (left panels) for specified heating simulations with (a) upper-level shear starting in the heating region, (b) low-level shear, and (c) low- and midlevel shear (solid lines). The thin dotted line in the left panels is the control simulation. Initial wind profiles corresponding to the simulations are shown in the right panels; thin horizontal lines mark the depth of the heating region.

waves with phase speeds between -10 and $5 \mathrm{~m} \mathrm{~s}^{-1}$ in the spectrum shown in Fig. 15a. These wave characteristics match the characteristics of waves generated by upper-level shear in squall line simulations (Fig. 8b), as well as the wave characteristics generated by the obstacle effect. The increase in the magnitude of eastward momentum flux due to the obstacle effects is quite small in the specified heating simulations just at this one frequency; however, one must keep in mind that in the squall line simulations, many more frequencies are present, and integrated over all the frequencies, the obstacle effect can change the magnitude of eastward momentum flux by up to $50 \%$ as shown in Fig. 6 d.

\section{d. Low-level shear}

The effects of a $15 \mathrm{~m} \mathrm{~s}^{-1}$ low-level westward jet are explored in a specified heating simulation with a shear layer ranging from the surface to the height of $2.5 \mathrm{~km}$. The initial wind profile is shown in Fig. $15 \mathrm{~d}$ and the resulting phase speed spectrum is shown in Fig. 15c. In this simulation westward $\bar{F}_{m}$ is increased and eastward $\bar{F}_{m}$ is decreased as compared to the control simulation.

The asymmetry between eastward and westward propagating waves in the case of low-level shear only is primarily due to the tilt of the paths along which parcels oscillate and is not very likely to be caused just by the change in wave vertical group velocities. In the specified heating simulation, the strong low-level shear tilts the paths along which parcels oscillate toward the west, and thus increases westward wave activity. This reasoning is consistent with the results from a model by Fovell et al. (1992) in which a westward-tilted mechanical oscillator produced an excess of westward- and a deficit of eastward-propagating waves as compared to a vertically oriented oscillator.

Comparison of Figs. 12 and $15 \mathrm{c}$ shows that wave characteristics from the simple heating runs, and the full squall line simulations for the case of only low-level shear, show some discrepancies. The simple heating simulations produce an excess of westward-propagating waves whereas the opposite kind of wave distribution is observed in the squall line. This is due to the opposite tilt of the paths along which parcels oscillate. In the squall line simulation the main updraft, and therefore the line along which parcels oscillate, is tilted eastward during the mature stage. This is because the tilt of the updraft in the squall line is not only determined by the low-level shear below $3 \mathrm{~km}$ but is also determined by the cold pool strength. In the squall line simulations in this study the lower level westward wind generates positive, counterclockwise, horizontal vorticity which counteracts the negative horizontal vorticity generated by the horizontal gradient of buoyancy induced by the presence of a cold pool. When the two vorticity production mechanisms are in exact balance, there is no tilt in the updraft (Rotunno et al. 1988). In the presence of a strong cold pool in the mature stages of the storm, this updraft is tilted to the east, explaining the excess of eastward-propagating waves. In the specified heating simulations, the westward shear simply tilts the paths along which parcels oscillate eastward. Therefore, since the tilt of the paths along which air parcels oscillate in the squall line is opposite that in the the simple heating model, the two simulations produce a dominance of waves propagating in opposite directions.

\section{e. Midlevel shear}

To complete the study, a $10 \mathrm{~m} \mathrm{~s}^{-1}$ eastward shear layer is added to the $2.5-\mathrm{km}$ low-level jet wind profile described in the above section. This wind profile and the corresponding momentum flux phase speed spectra for the simulation are shown in Figs. $15 \mathrm{f}$ and $15 \mathrm{e}$ respectively. Compared to the run with only a $2.5-\mathrm{km}$ deep low-level jet (Fig. 15c), there is a slight increase in westward momentum flux and a strong decrease in eastward momentum flux.

The $10 \mathrm{~m} \mathrm{~s}^{-1}$ midlevel shear eliminates eastward- 
propagating waves with phase speeds between 0 and 10 $\mathrm{m} \mathrm{s}^{-1}$. A similar phenomena was noted in the distribution of waves generated by the squall lines. Thus, the filtering mechanism is indeed present in the lower part of the troposphere. A shear layer located within the heating region inhibits the propagation of gravity waves with phase speeds equal to the wind speed in the shear layer just as the shear layer at the top of the heating region did.

Westward momentum does not change significantly in the presence of midlevel shear. There is little evidence of wave generation via the obstacle effect in this midlevel shear simulation. The wind changes only by $10 \mathrm{~m}$ $\mathrm{s}^{-1}$ in a shear zone that is mostly confined within the heating region. In order to produce a pronounced obstacle effect, strong flow over the heating region is necessary. This agrees with the findings from squall line simulations, in which increased midlevel eastward shear did not substantially increase the momentum flux of westward-propagating waves.

\section{Summary and discussion}

In this study, numerous two dimensional simulations of tropical squall lines were performed with different vertical profiles of the horizontal wind. The horizontal wind shear was varied in the three layers of the troposphere shown in Fig. 1, and the effects of these changes on the structure of gravity waves propagating into the stratosphere were examined. The primary measures of changes in the wave activity were the momentum flux magnitudes and phase speed spectra of eastward and westward propagating gravity waves at the height of $15 \mathrm{~km}$. Since the structure of stratospheric gravity waves showed a strong temporal variation, momentum flux calculations were performed separately for different stages of the squall line development to show that the observed differences were not an artifact of the selected analysis time. To verify speculations about the interaction of wind shear with a thermal forcing, simplified simulations using a specified heating region as a wave generator were compared to the squall line simulations.

The following two sections summarize how tropospheric wind shear affects convective gravity wave generation, and explain why tropospheric wind shear is a parameter that should be included in future parameterizations of convectively generated gravity waves in GCMs.

\section{a. Wave-generation mechanisms}

The squall line simulations and the specified heating simulations suggest that the vertical extent of the heating determines the dominant vertical wavelength of the generated waves, and hence by (10), also determines the dominant range of the intrinsic phase speeds at the wave-generation level. The phase speed spectrum of waves propagating into the stratosphere is then modified by the vertical profile of the horizontal wind.

Low-level shear acts mainly to create an asymmetry between eastward- and westward-propagating waves, without changing the dominant range of wave phase speeds. In a squall line simulation with a westward lowlevel jet, the stratospheric eastward momentum flux of simulated gravity waves was much greater than the westward momentum flux. This asymmetry is due to the enhancement of wave propagation in the direction in which the main storm updraft is tilted as described in Fovell et al. (1992).

Both the specified heating and full squall line simulations show that any shear layer above $2.5 \mathrm{~km}$ has three effects on the generated wave spectrum: it acts to reduce the stratospheric momentum flux of waves propagating in the same direction as the storm-relative mean wind in the shear zone, it can increase the momentum flux of waves propagating opposite to the storm-relative mean wind, and it alters wave group velocity, which can effectively change the observed wave momentum flux above the wave source.

Momentum flux phase speed spectra show that the decrease in wave propagation due to a shear layer located in the troposphere occurs in a manner similar to critical-level filtering. Waves with phase speeds equal to the storm-relative mean wind in the shear layer are filtered out and are not present in the stratosphere.

Shear near the top and just over the heating region can generate an excess of waves propagating opposite to the storm-relative flow in a manner similar to the obstacle effect. These waves have relatively low frequencies, $\omega \leq 0.01$ cycles per minute, and low horizontal wavenumbers, $k \leq 0.0075$ cycles per kilometers. The momentum flux of waves generated in this fashion is very sensitive to the location of the shear layer with respect to the heating region. The obstacle effect is not present when the wind shear layer is located strictly within the heating region rather than near the top and above it. These results are consistent with the study of Clark et al. (1986), who found that shear going over boundary layer convection produced a greater wave response than the same strength shear going through the convection. The momentum flux carried by waves generated by the obstacle effect also decreases when the shear layer is moved too high above the heating region.

Kershaw (1995) also found that increased flow through and over a convective element increases the wave response. Momentum flux estimates in his study are, however not easily comparable to the present work, because wave fluxes with opposite sign were not shown separately, but were added together.

Third, vertical wind shear significantly alters the vertical group velocity of convectively generated gravity waves, and therefore alters both the propagation time from source to analysis level, as well as the amount of damping waves undergo before reaching the analysis level. This factor should be taken into account when 
inferring gravity wave-generation mechanisms both from numerical models as well as observations.

Effects of shear in the upper level of the troposphere were previously examined by Lane et al. (2001). Their initial zonal wind profile contained a $5 \mathrm{~m} \mathrm{~s}^{-1}$ westward shear layer located from 14 to $16 \mathrm{~km}$. Through their gravity wave source tensor analysis, the authors found the upper shear layer not responsible for any wave generation and concluded that shear was not important in the gravity wave-generation process. The analysis presented in this paper suggests that indeed the upper shear layer used in Lane et al. (2001) would have little effect on wave generation: $5 \mathrm{~m} \mathrm{~s}^{-1}$ is not enough wind shear to produce a pronounced obstacle effect especially since the shear is located far above the main heating region. However, based on the current study, their latter conclusion seems valid for their particular wind profile only.

\section{b. Implications for GCMs}

Typically, GCMs have only included gravity wave parameterizations appropriate for topographic gravity waves due to the lack of an accurate understanding of convective sources. Those that do attempt to parameterize convectively generated gravity waves tend to assume that tropospheric convection generates gravity waves isotropically, carrying equal momentum flux in all directions (Rind et al. 1988). The simulations performed in this study demonstrated that this assumption is most likely erroneous. Chun and Baik (1998) describe a parameterization that generates waves anisotropically assuming constant wind flowing over a steady heat source. The waves generated in this parameterization are all stationary, like topographic waves, but very unlike the waves generated in our squall line simulations.

For most tropospheric wind profiles considered, the distribution of stratospheric gravity waves did not show east-west symmetry. One measure of wave asymmetry is the ratio of eastward to westward momentum flux carried by waves generated in the troposphere. This ratio for the low-, middle-, and upper-level shear simulations averaged over the three stages of storm development together is shown in Fig. 16. Figure 16 shows that, to a first-order approximation, the ratio of eastward to westward momentum flux is dependent only on the storm-relative mean wind, regardless of the details of the tropospheric wind profile. This figure also shows that the generated wave field is seldom symmetric in the east-west direction; gravity waves propagating rearwards relative to the storm usually dominate.

\section{Conclusions}

Gravity waves generated by convection are generated throughout the depth of the troposphere. Most of the wave generation occurs in the region of latent heat release, however some waves are generated by flow near the top and over the heating region. Dominant features

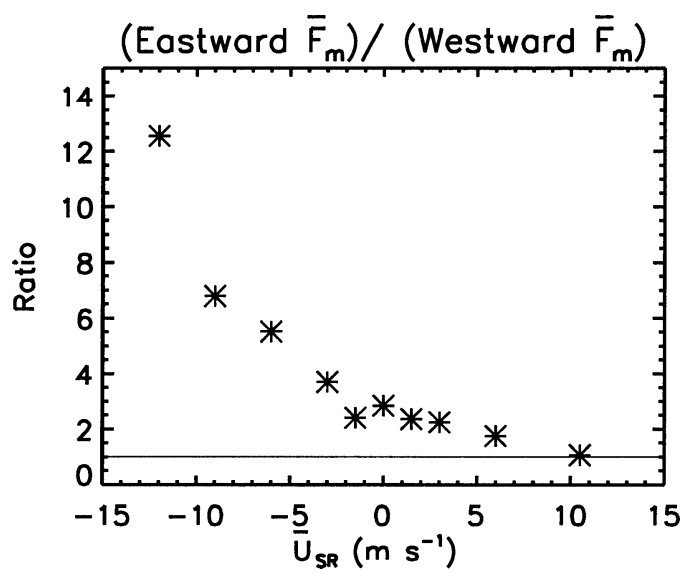

FIG. 16. Ratio of the domain-averaged eastward-to-westward momentum flux averaged over all stages of storm development vs storm relative mean wind $\bar{U}_{\mathrm{SR}}$ for simulations with shear varying in the three layers of the troposphere. The thin horizontal line marks the ratio of 1 (when eastward and westward propagating waves are generated symmetrically).

of the phase speed spectrum of convectively generated waves are determined by the vertical scale of the heating region, and are modified by the vertical structure of the horizontal wind.

Shear in the upper layer of the troposphere has the greatest effect on the wave distribution since it acts to diminish the momentum flux of gravity waves propagating in the same direction as the stormrelative mean wind and it increases the momentum flux of gravity waves propagating opposite to the storm-relative mean wind. The decrease in wave momentum flux occurs in a manner similar to critical-level filtering, whereas the wave generation is caused by the obstacle effect.

Tropospheric wind can also strongly refract waves generated by the heating region. Although the momentum flux of waves generated in the troposphere is not always altered by the shear, a decrease in momentum flux may be observed in the stratosphere. This effect is due to the fact that some of the refracted waves have very small group velocities, and thus require a long time to reach the stratosphere, and suffer from more damping.

Finally, the study has shown that tropospheric wind shear should be included in future gravity wave parameterizations. For most tropospheric wind profiles, gravity waves generated by tropical convection show a strong asymmetry between the eastward- and westwardpropagating waves. To the first-order approximation, this asymmetry can be determined by the wind relative to the storm above the tropospheric heating region.

Acknowledgments. This research was supported by the National Science Foundation NSF Grant ATM9979241 and the National Aeronautics and Space Administration NASA Grant NAG-1-2193. 


\section{REFERENCES}

Alexander, M. J., 1998: Interpretations of observed climatological patterns in stratospheric gravity wave variance. J. Geophys. Res., 103 (D8), 8627-8640.

— stratosphere over convection. Geophys. Res. Lett., 22, 20292032.

— , and J. Holton, 1997: A model study of zonal forcing in the equatorial stratosphere by convectively induced gravity waves. J. Atmos. Sci., 54, 408-419.

_ and R. A. Vincent, 2000: Gravity waves in the tropical lower stratosphere: A model study of seasonal and interannual variability. J. Geophys. Res., 105 (D14), 17 983-17 993.

_ J. R. Holton, and D. R. Durran, 1995: The gravity wave response above deep convection in a squall line simulation. $J$. Atmos. Sci., 52, 2212-2226.

— J. H. Beres, and L. Pfister, 2000: Tropical stratospheric gravity wave activity and relationships to clouds. J. Geophys. Res., 105 (D17), 22 299-22 309.

Allen, S. J., and R. A. Vincent, 1995: Gravity wave activity in the lower atmosphere: Seasonal and latitudinal variations. J. Geophys. Res., 100 (D1), 1327-1350.

Canziani, P. O., J. R. Holton, E. F. Fishbein, and L. Froidevaux, 1995: Equatorial Kelvin wave variability during 1992 and 1993. J. Geophys. Res., 100, 5193-5202.

Chun, H. Y., and J. J. Baik, 1998: Momentum flux by thermally induced internal gravity waves and its approximation for largescale models. J. Atmos. Sci., 55, 3299-3310.

Clark, T. L., T. Hauf, and J. P. Kuettner, 1986: Convectively forced internal gravity waves: Results from two-dimensional numerical experiments. Quart. J. Roy. Meteor. Soc., 112, 899-925.

Dunkerton, T. J., 1997: The role of gravity waves in the quasi-biennial oscillation. J. Geophys. Res., 102 (D22), 26 053-26 076.

Durran, D. R., 1999: Numerical Methods for Wave Equations in Geophysical Fluid Dynamics. Springer-Verlag, 465 pp.

_ , and J. B. Klemp, 1983: A compressible model for the simulation of moist mountain waves. Mon. Wea. Rev., 111, 2341-2361.

Fovell, R., D. Durran, and J. R. Holton, 1992: Numerical simulations of convectively generated stratospheric gravity waves. J. Atmos. Sci., 49, 1427-1442.

Hitchman, M. H., and C. B. Leovy, 1988: Estimation of the Kelvin wave contribution to the semiannual oscillation. J. Atmos. Sci., 45, 1462-1475.

Holton, J. R., and R. S. Lindzen, 1972: An updated theory for the quasi-biennial cycle of the tropical stratosphere. J. Atmos. Sci., 29, 1076-1080.

Kershaw, R., 1995: Parametrization of momentum transport by convectively generated gravity waves. Quart. J. Roy. Meteor. Soc., 121, 1023-1040.
Lane, T. P., M. J. Reeder, and T. L. Clark, 2001: Numerical modeling of gravity wave generation by deep tropical convection. J. Atmos. Sci., 58, 1249-1274.

McLandress, C., M. J. Alexander, and D. L. Wu, 2000: Microwave limb sounder observations of gravity waves in the stratosphere: A climatology and interpretation. J. Geophys. Res., 105 (D9), $11947-11967$.

Pandya, R. E., and M. J. Alexander, 1999: Linear stratospheric gravity waves above convective thermal forcing. J. Atmos. Sci., 56, 2434-2446.

Percival, D. B., and A. T. Walden, 1993: Spectral Analysis for Physical Applications. Cambridge University Press, 583 pp.

Pfister, L., and Coauthors, 1993: Gravity waves generated by a tropical cyclone during the STEP tropical field program: A case study. J. Geophys. Res., 98 (D5), 8611-8638.

Piani, C., D. Durran, M. J. Alexander, and J. R. Holton, 2000: A numerical study of three-dimensional gravity waves triggered by deep tropical convection and their role in the dynamics of the QBO. J. Atmos. Sci., 57, 3689-3702.

Ray, E. A., M. J. Alexander, and J. R. Holton, 1998: An analysis of structure and forcing of the equatorial semiannual oscillation in zonal wind. J. Geophys. Res., 103 (D2), 1759-1774.

Rind, D., R. Suozzo, N. K. Balachandran, A. Lacis, and G. Russell, 1988: The GISS global climate-middle atmosphere model. Part I: Model structure and climatology. J. Atmos. Sci., 45, 329-370.

Rotunno, R., J. B. Klemp, and M. L. Weisman, 1988: A theory for strong, long-lived squall lines. J. Atmos. Sci., 45, 463-485.

Salby, M. L., and R. R. Garcia, 1987: Transient response to localized episodic heating in the Tropics. Part I: Excitation and short-time near-field behavior. J. Atmos. Sci., 44, 458-498.

Sato, K., 1992: Vertical wind disturbances in the afternoon of midsummer revealed by the MU-radar. Geophys. Res. Lett., 19, 1943-1946.

_ 1993: Small-scale wind disturbances observed by the MU radar during the passage of typhoon Kelly. J. Atmos. Sci., 50, 518537.

Skamarock, W. C., and J. B. Klemp, 1993: Adaptive grid refinement for two-dimensional and three-dimensional nonhydrostatic atmospheric flow. Mon. Wea. Rev., 121, 788-804.

Takahashi, M., and B. A. Boville, 1992: A three-dimensional simulation of the equatorial quasi-biennial oscillation. J. Atmos. Sci., 49, 1020-1035.

Vincent, R. A., and M. J. Alexander, 2000: Gravity-waves in the tropical lower stratosphere: An observational study of seasonal and interannual variability. J. Geophys. Res., 105 (D14), 17971 17982.

Wu, D. L., and J. W. Waters, 1996: Satellite observations of atmospheric variances: A possible indication of gravity waves. Geophys. Res. Lett., 23, 2631-3634. 